Gerión. Revista de Historia Antigua

ISSN: 0213-0181

http://dx.doi.org/10.5209/geri.68589

\title{
Il funus imperatorum ed il "Teatro del potere". Le esequie di Costantino e la reinvenzione dei riti funerari imperiali in un cosmo che progressivamente si fa cristiano
}

\author{
Antonio Pio Di Cosmo ${ }^{1}$
}

Recibido: 11 de febrero de 2019 / Aceptado: 7 de septiembre de 2019

Riassunto. Il contributo analizza il ruolo giocato dalla cultura materiale della regalità nel funus imperatorum. Questa ricerca applica le conoscenze in materia archeologica, antropologica e storica, per raccontare l'azione della corte imperiale, che risolve le questioni circa i problemi di rappresentazione del funerale imperiale. In questo senso si vagliano le modalità di spettacolarizzazione delle strategie di comunicazione che modellano i protocolli degli imperatori romani del Tardoantico.

Parole chiave: funus imperatorum; Costantino; "Teatro del potere"; esequie cristiane.

\section{[en] The Funus Imperatorum and the "Theatre of Power". The Obsequies of Constantine and the Reinvention of Imperial Funeral Rites in a World that gradually Becomes Christian}

\begin{abstract}
This contribution analyzes the role of the material culture of royalty during the funus imperatorum. This inquiry applies archaeological, anthropological and historical effectiveness and reports them to the work of sovereigns' entourage, which concludes questions about the representation problems in the imperial funeral. In this way, it scrutinizes the spectacularization of communication strategies, that model etiquette of Roman emperors in Late Antiquity.

Keywords: Funus Imperatorum; Constantine; "Theatre of Power"; Christian Funeral.
\end{abstract}

Sommario: 1. Introduzione. 2. Il copione: il protocollo per il rito di "espulsione dalla società". 3. Il protagonista: l'imperatore defunto da consegnare al cielo. 4. Gli attori "coprotagonisti": la corte ed i partecipanti. 5. Il palcoscenico: i SS. Apostoli ed il culmine del funus. 6. Gli oggetti di scena: la veste ed il sepolcro di porfido. 7. Conclusioni. 8. Bibliografia.

Cómo citar: Di Cosmo, A. P. (2020): Il funus imperatorum ed il "Teatro del potere". Le esequie di Costantino e la reinvenzione dei riti funerari imperiali in un cosmo che progressivamente si fa cristiano, en Gerión 38/1, 157-192. 


\section{Introduzione}

La presente ricerca procede dalla spettacolarizzazione delle ritualità che concernono i "riti di espulsione dalla società" degli imperatori romani fra III e V sec., ${ }^{2}$ riti che vengono ad essere reinventati a seguito delle esequie di Costantino, che fungono da catalizzatore rispetto alla prassi tradizionale. L'insieme di pratiche, che costituiscono il funus imperatorum inscenato a Bisanzio, lascia sopravvivere entro un contesto dominato dal cristianesimo la maggior parte del patrimonio immateriale romano concernente il lutto ed il suo tempo. Un cerimoniale collettivo dunque, che al di là della manipolazione rituale della salma imperiale, costituisce piuttosto un momento di riaffermazione ed autoriconoscimento dell'intera comunità. Il rito allora si indirizza ai singoli partecipanti, che vengono meglio 'assorbiti' attraverso gli espedienti del "Teatro del potere", entro il corpo politico dell'Impero. Siamo di fronte ad un insieme di stratagemmi che, in fin dei conti, avvantaggia l'Istituzione monarchica e soprattutto la rafforza attraverso un processo di costruzione della memoria in chiave retorica.

Un rito costruito dalla corte che fa tesoro dell'esperienza maturata durante il funerale costantiniano. Questa ha ad operare attraverso l'uso di un linguaggio visivo incisivo ed aggrega i momenti salienti del cerimoniale attorno ad un canovaccio, che dopo la 'reinvenzione' della prassi per il primo imperatore definitosi apertamente cristiano, si è cristallizzato. Momenti standardizzati che vanno a costituire un canone, utile ad accreditare, se ricalcato fedelmente, l'insieme di atti cerimoniali celebrati nelle future esequie imperiali.

Alla luce di ciò è possibile analizzare il funus a guisa di una vera e propria forma di spettacolo, che prevede un copione: il protocollo; un protagonista quale l'imperatore defunto e consegnato ai celesti; degli attori "coprotagonisti" interpretati dai 'figuranti' della pompa funebre e dai partecipanti che vengono coinvolti in diverso modo; un palcoscenico come Costantinopoli, la "Città regina" con il punto focale dell'Apostoleion dove gli augusti vengono inumati e, infine, degli oggetti di scena che ottimizzano l'efficacia affabulatoria dello spettacolo, fra cui spiccano la veste funebre ed il sepolcro.

\section{Il copione: il protocollo per il rito di "espulsione dalla società"}

Il funus imperatorum si costruisce come un insieme di moduli spiegati entro un codice cerimoniale, modellato in formule aperte. Moduli che pongono in dialogo la corte che progetta il rito ed i fruitori della cerimonia, i quali sono inseriti in una 'magmatica' e 'polifonica' situazione materializzata innanzi ai propri occhi. La corte pone in essere un esercizio fenomenologico insomma, capace di rendere gli astanti dei partecipanti. Lo struttura poi come un 'rito aperto', dove 'figuranti' e partecipanti hanno la possibilità di alterare il protocollo-base, cosa che rende il medesimo ordo elastico ed altamente permeabile alle sollecitazioni esterne. Una struttura in continuo aggiornamento dunque, passibile di innovazioni e capace di accogliere alcuni dei comportamenti non strutturalizzati.

Perea Yébenes 2005, 118. 
Il protocollo ha la capacità di fissare le pratiche commemorative in un insieme di immagini montate in progressione, unite da una struttura processuale, che non ammette un'unica prospettiva, ma tante prospettive integrate e, persino, diverse angolazioni; tante quante quelle dei fruitori del rituale. Il rito si dimostra così unico e molteplice. All'opera di costruzione delle suggestioni visive da parte della corte, si oppongono le differenti impressioni dell'osservatore, che configurano a loro volta vere e proprie "istantanee" del rito. La cerimonia può così essere smontata in una sequenza di immagini, generando un vero e proprio "circuito visivo" autoreferenziale, che è frutto della percezione ottica del singolo osservatore. Le esequie pertanto possono essere interpretate come un percorso volto alla scoperta della sintassi che lega le singole immagini e le suggestioni suscitate dalla trama del rito. Un processo di invenzione insomma, che plasma la realtà entro un modello narrativo efficace e "negoziato" con lo sguardo dei partecipanti.

Al contempo, la spettacolarizzazione del lutto imperiale investe la popolazione, che attraverso la trama del protocollo si ricostruisce come "comunità di immaginazione", entro un processo di ricompattazione del corpo sociale. ${ }^{3}$ Pratiche volte a coinvolgere, come quella dello iustitium: sospeso il "tempo ordinario" ed i negotia, si permette al popolo di partecipare alle cerimonie del tempo funesto dell'Impero.

Il protocollo poi tenta di sistematizzare i comportamenti degli uomini che si relazionano con l'evento morte e partecipano alle scene corali costruite dai riti funerari. $\mathrm{Li}$ immette entro gli "spazi ordinati" delle prossemiche accolte nel cerimoniale che scandiscono il racconto del tempo funesto, sistematizzandole secondo precise cadenze di "tagli scenici", che rinviano ad "una metafisica idea di teatro". ${ }^{4}$ Il rito pertanto ci mette di fronte ad un'immediata ed, altresì, mediata rappresentazione delle qualità paradigmatiche dell'evento morte. Mentre i prodotti della cultura materiale come la veste funebre, il fercolo ed, infine, il sepolcro in porfido o altro marmo pregiato fungono da indispensabile anello di congiunzione fra le istanze di chi concepisce la sequenza dei gesti e la prassi in sé. La catalogazione delle gestualità, che realizza un repertorio esemplare, consente di ridurre a schema dimostrativo le possibili opzioni prossemiche. Queste vengono poi stereotipate, per assumere funzione normativa, quali 'garanzie materiali' della dignità del defunto. Eppure oltre la patina aulica sopravvive un' esperienza umanissima, che non mortifica né il "contenuto umano", né l'emozionalità dell'evento. ${ }^{5}$ La regolarizzazione costituisce piuttosto la sublimazione e la teatralizzazione dell'evento doloroso, realizzata a mezzo dell'uso costante di emblemi che lo allontanano dal reale.

La devoluzione di beni di pregio all'effimero fa parte di quell'attitudine alla narrazione fatta propria dall'Istituzione imperiale, specie allorché si sente minacciata dalla frattura causata dall'evento morte. Questa sente la necessità di ribadire attraverso il fasto esibito il proprio primato gerarchico d'origine divina. Un dispendio che non è indirizzato all'autocompiacimento della corte, ma deve servire piuttosto a coattare l'animo ed il comportamento dei fruitori dello spettacolo. Tale meccanismo è volto ad agevolare il fruitore che può meglio calarsi nel tempo del dolore, in qualità di vero e proprio "attore", adeguando la sua condotta al registro comportamentale proprio del rito. Il fruitore accetta così di vivere uno status che è "provvisorio" e tutto interno alla dialettica della celebrazione, mentre racconta la storia in qualità di attore

Alvar 1999, 272-280; Parisi 2010, 108.

Panascià 1993, 9-32; Resta 2010, 21-45.

Panascià 1993, 9-32. 
"coprotagonista". 6 Questi appare coattato dal "mettersi in mostra" dell'Istituzione imperiale posta di fronte all' evento morte e dall'azione prossemica stimolata dalla corte e catalizzata dagli altri 'coattori-partecipanti'. Una partecipazione incoraggiata anche a livello sensorio attraverso il corredo materiale della cerimonia funebre: le luci tremolanti delle fiaccole, i suoni mesti, la presenza di innumerevoli ecclesiastici salmodianti. Una coattazione ottenuta dal protocollo ed orientata da una consapevolezza: l'esser parte del rito che prende corpo è fondamentale, non solo perché "le cose accadono" ma, in vero, poiché vale la pena parteciparvi in ragione del 'liturgico stupore' provato innanzi al dispiegarsi di tutti quei simboli. ${ }^{7}$

Il protocollo orbene pone delle problematiche di rappresentazione ideologica. Queste devono essere comprese alla luce dell'intersoggettività che caratterizza le interazioni e fornisce di significatività ideologica e culturale la prossemica propria del rito. Il funerale imperiale può così costituire un'occasione privilegiata per dispiegare con maggior potenza la "retorica e la poetica dello sguardo" entro scene orientate dalle finalità politiche della "voce narrante" della corte organizzatrice. ${ }^{8}$ Un punto di vista, quello della corte, che è fondamentale per comprendere le ragioni che costruiscono la pompa funebre per quel che è, con la sua lunga sfilata di 'partecipantifiguranti', portatori al contempo delle istanze e dei simboli degli ordini sociali. Siamo di fronte alla messa in scena per immagini di relazioni sociali complesse, che sono alla base del "patto sociale", la cui tensione deve necessariamente essere sublimata entro i confini del dolore ritualmente richiesto dal tempo del lutto.

Costoro abbigliati con le insegne del rango ed abiti suntuosi realizzano una teatralizzazione del corpo sociale, mentre l'ostentazione rituale della ricchezza costituisce una "scenografia del meraviglioso", che ha come cifra l'abbondanza declinata nel segno dell'effimero. La messa in scena del funus imperatorum attualizza una necessità tutta culturale, che si realizza più tardi entro lo spazio delle relazioni della società romano-orientale, laddove si "reificano la coscienza della gerarchia del cosmo (...) nella gestualità dell'etichetta, nei segni, nella musica". ${ }^{9}$ L'occupazione dello spazio attraverso il rito funebre rientra poi tra quegli espedienti utili a ribadire il controllo sociale dell' Istituzione imperiale, quale espressione di un ordine che non è alterabile culturalmente, ma unifica gli "attori" dell'azione entro un antropocosmo.

Uno "spazio sospeso", quello del cerimoniale connesso al consumarsi del funus, che ha un ulteriore scopo: deve far irrompere il meraviglioso in questo momento di rottura, perché con questi riti si deve consegnare al cielo l'imperatore. Il funerale permette di immaginare la sua ultravita, quale poziore realtà resa più percepibile da una fictio ideologica. Al contempo, segna l'avvicendarsi del nuovo reggente pro tempore, proclamando piuttosto l'aeternitas imperii.

\section{Il protagonista: l'imperatore defunto da consegnare al cielo}

Il funus imperatorum si costruisce attorno alla persona dell'imperatore defunto ed inserisce entro il contesto aulico del protocollo i consueti riti di "manipolazione

\footnotetext{
Resta 2010, 21-45.

Resta 2010, 21-45.

Resta 2010, 21-45.

Panascià 1993, 9-32; Carile 2003, 589-656.
} 
del cadavere" finalizzati "all'espulsione dalla società". ${ }^{10} \mathrm{E}$ sebbene questi è effettivamente assente a causa della sua dipartita, appare più che presente, in quanto è il protagonista del rito che deve collocarlo entro i celesti. Tutto il cerimoniale si obera del compito di persuadere il pubblico, fin a convincerlo a credere ad una realtà più grande: il proprio augusto è immortale, perché fatto partecipe del "mistero della regalità". ${ }^{11}$ Siamo di fronte ad una realtà sempre difficile da perorare, ma essenziale tanto per la credibilità dell'Istituzione, quanto per la sua sopravvivenza. Tale credenza appare pure funzionale a suturare quelle lesioni intercorse nella continuità di gestione dell'Impero in ragione della dipartita del suo rappresentante pro tempore. L'acquisizione dello stato divino prima e di un posto tra i celesti poi per l'imperatore sono ascrivibili a due tentativi volti a 'blindare' l'istituzione imperiale, allorché si raffronta con l'evento morte. Il rito pertanto deve organizzarsi attorno ad una sommessa finalità: evocare quel che può essere definito il 'doppio' corpo facente capo all'augusto. Sulla scorta dei dati raccolti dalle narrazioni delle esequie imperiali si ha a sostenere che all'imperatore romano può essere additato un primo corpo, che è quello fisico, mortale e "privato", il quale subisce i benefici del fuoco attraverso la cremazione rituale della pira funeraria. Alle spoglie corrisponde un secondo corpo: metafisico, "politico" e "pubblico" a cui gli imperatori hanno accesso dopo questi riti volti alla purificazione della salma. ${ }^{12} \mathrm{Al}$ contempo, il Senato, quale sommo organo religioso dell'Impero, esercita la facoltà di fare dell'imperatore un dio attraverso la lex sacrata, quale atto formale, costitutivo e pubblico certificante il raggiungimento della natura divina, che viene votato con senatoconsulto. ${ }^{13}$ Tale legge che autorizza la consecratio fa derivare particolari diritti in capo al defunto, il quale oltre a vantare il titolo di divus, cioè di uomo divinizzato, diviene destinatario di un culto da officiarsi, può essere titolare di un tempio, nonché riceve dei sacerdoti per le celebrazioni. ${ }^{14}$ Lo status divino conferito a tramite di un giudizio tutto umano, come quello dei senatori, ha molte implicazioni. Sottoporre il defunto imperatore alla valutazione dei senatori significa inserire una 'clausola di salvaguardia', quale "ulteriore espressione della libertà di Roma", ${ }^{15}$ entro il contesto della "libera competizione politica di un'aristocrazia di eguali". ${ }^{16}$ Pertanto il substrato religioso costituisce solo uno dei componenti di un atto ufficiale, che ha piuttosto natura politica e conserva solo un qualche legame col rito di consecratio officiato dal pontefice e da un magistrato e destinato a conferire sacralità. ${ }^{17}$ Appiano poi precisa i limiti dell'operabilità dell'istituto: la legge viene emanata se l'augusto non ha "governato in modo tirannico" o non sia "stato degno di disprezzo". ${ }^{18}$

Per poter dimostrare a livello visivo tale finalità il "rito di espulsione dalla società" dell'augusto deve accogliere al suo interno elementi tratti dal trionfo. ${ }^{19}$

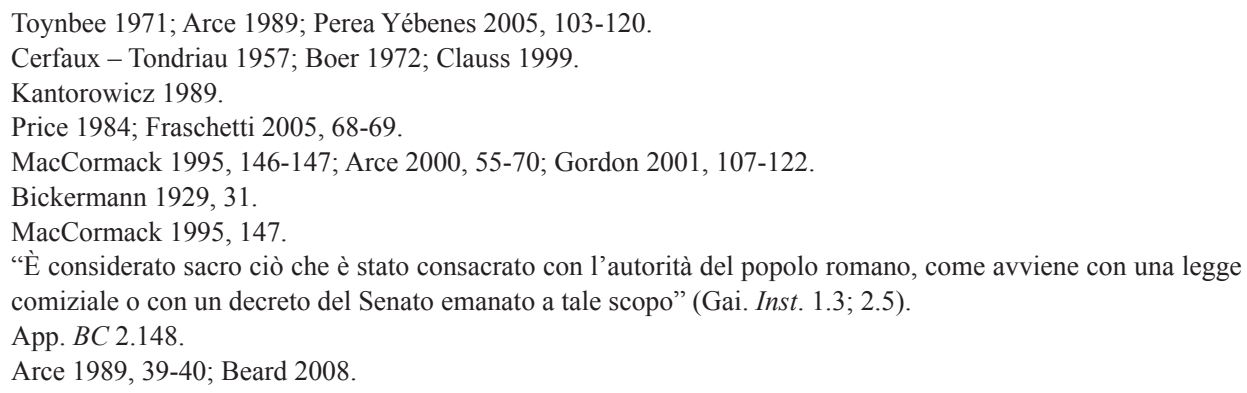


Componenti che non devono sovrastare lo spazio concesso al dolore, a maggior ragione se pubblico. Asinio Gallo e Lucio Arrutino propongono di immettere nel funerale di Ottaviano elementi espliciti del rito di trionfo: una processione funebre aperta dalla statua della Vittoria, posta in Senato, e il passaggio del corteo sotto la Porta detta Triumphalis. ${ }^{20}$ Stupisce ancora un'innovazione fondamentale nei riti funerari romani: il cadavere, oggetto impuro per eccellenza, eccezionalmente può essere condotto ed esposto intra muros, persino in luoghi pubblici come basiliche e templi. L'oggetto tanto venerato, difatti non è una salma, ma la 'reliquia' di un divus e dinnanzi ad un dio cadono i tabù degli uomini. ${ }^{21} \mathrm{E}$ se il problema connesso all'impurità di un cadavere viene facilmente risolto nelle città di provincia, non può accadere lo stesso a Roma. Occorre allora un'apposita adeia, per quei soggetti quali Tiberio, che sono venuti a contatto con la salma ed hanno la necessità di entrare in spazi consacrati come il Senato. Ottaviano è dunque rappresentato come un dio, ma l'élite repubblicana non sembra crederci troppo. Le elaborazioni culturali concernenti la tradizionale impurità della morte sono molto difficili da superare.

Per rendere più credibile il transito di natura, si ricorre poi ad un espediente giuridico: un testimone. Numerio Attico attesta sotto giuramento di aver visto l'anima di Ottaviano sollevarsi dalla pira ed ascendere al cielo. Questo intervento, che gli guadagna un milione di sesterzi donatigli da Livia, vuole riempire quei vuoti creati da un procedimento che a Roma non solo può essere considerato nuovo, ma presenta alcune difficoltà ad attecchire. La divinizzazione imperiale ed il relativo culto si pongono poi quale tassello fondamentale del nuovo corso inaugurato dall'istituto del principato.

Il rito funereo cittadino, che prevede la processione per la Via Sacra ed almeno un'orazione, ${ }^{22}$ si conclude infine al campo di Marte, laddove il corpo del defunto prende posto sulla pira rituale per essere protagonista di un rito di purificazione, che consta nell' incenerimento del cadavere. ${ }^{23}$ Una pratica nata con caratteri sanitari, che viene reinterpretata dalla propaganda e diviene un mezzo per garantire il raggiungimento di quello stato divino che ha suscitato non poche perplessità.

Il tema del funus imperatorum solitamente viene liquidato in modo veloce dagli storici, che parlano meramente di un funus publicum nei casi di esecuzione di ritualità convenienti per il rango imperiale. ${ }^{24}$ Per Tiberio Svetonio riferisce: crematumque publico funere. ${ }^{25}$ Altrettanto velocemente viene raccontato il funerale di Claudio. Tacito vi aggiunge un dettaglio non trascurabile, perché afferma che i funerali sono celebrati in modo simile a quelli di Augusto: (...) et funeris solemne perinde ac divo Augusto celebratur. ${ }^{26}$ Un fasto che viene confermato dall' Apokolokyntosis, che ironizza sulla presenza del corteo di musici come corollario di un rito conveniente ad un imperatore. ${ }^{27}$ Nerva viene seppellito con il fasto consueto e si celebra un funerale

20 Tac. Ann. 1.8.4-5; Suet. Aug. 100; Taylor 1931; Richard 1966a, 66-78; 1966b, 313-325; 1966c, 784-804; 1966d, 127-142, e 1978, 1121-1134; Huntington - Metcalf 1979, 46; Arce 1989, 39; Crook 1996, 113-146; Lozano 2002, e 2007, 139-152; Nogales - González 2007.

21 Suet. Aug. 100-101; D.C. 56.31.1.

22 Arce 2000b.

23 Suet. Aug. 100.3; Coarelli 1983, e 1985, 308.

24 Wesch-Klein 1993.

25 Suet. Tib. 75.3.

26 Tac. Ann. 12.3.

27 Sen. Apocol. 13; Alvar - Lozano 2008, 35-56. 
simile a quello di Augusto: Cuius corpus a senatu, ut quondam Augusti, honore delatum in sepulcro Augusti sepultum est. ${ }^{28}$

Adriano, morto in odio a tutti, addirittura viene sepolto in un primo tempo fuori Roma. Un rito privato che non può essere celebrato con il solito cerimoniale e, pertanto, lascia solo un vago ricordo nelle fonti. ${ }^{29}$

Altrettanto poco sappiamo dei funerali di Antonino Pio, che muore nella sua villa di Lorium prossima a Roma. La formula: Decreti etiam sunt omnes honores, qui optimis principibus ante delati sunt, prova la celebrazione del funerale pubblico con tutti gli onori. ${ }^{30}$

Altri imperatori invece vengono deposti dal tumulto. La casistica delle loro esequie viene connotata da un cerimoniale inadeguato. I particolari "riti d'espulsione" approntati per quegli imperatori, privi del fasto consueto, evocano la damnatio che investe i loro protagonisti, ${ }^{31}$ tanto che si possono definire quali 'non' rito. Ciò accade, tra i molti, a Nerone e Domiziano. Il primo suicidatosi vede le sue ceneri deposte in un'urna, che nonostante tutto è degna dello status imperiale. ${ }^{32}$ Per il secondo si appronta un funerale definito nelle fonti "disonorevole" e la pira di fortuna viene composta dalla serva Fillis. Le ceneri dell'imperatore vengono collocate sempre dalla stessa serva nel Templum Gentis Flaviae e commiste a quelle della nipote Giulia, figlia di Tito. ${ }^{33}$ Lo stesso accade per Commodo che, vittima di una congiura, viene 'espulso' dalla città come se fosse un oggetto; questo è avvolto in una coperta e messo su di un carretto. ${ }^{34}$ Caracalla poi, morto in una congiura ordita da Macrino, viene cremato velocemente..$^{35}$ Eliogabalo invece viene gettato al Tevere dal ponte Emilio $^{36}$ o presso una cloaca. ${ }^{37}$

Ben più tardi Erodiano, narrando la prassi funeraria eseguita per Settimio Severo, fornisce un'informazione molto interessante: perdura la consuetudine della processione funebre e della pira, a cui si aggiunge il rito della liberazione di un'aquila; questa deve offrire al pubblico l'illusione che l'anima imperiale si sia tramutata in quell'animale..$^{38}$ Sotto questa forma pare più credibile che l'imperatore possa ascendere al cielo. Siamo di fronte ad un abile espediente che, evocando la metamorfosi dell'anima dell'imperatore nel volatile, ha ad affermare innanzi ad un più ampio pubblico l'effettivo realizzarsi dell'apoteosi. Ciò è a maggior ragione credibile, se si considera la descrizione del funerale di Pertinace, che precede quello di Settimio Severo. La trama del rito, che declina la particolare fattispecie del funus imaginarium, ${ }^{39}$ dimostra la necessità sentita dall'Istituzione imperiale di dover ribadire anche visivamente l'effettività della divinizzazione dell'imperatore. Il racconto, che introduce il volo al cielo dell'aquila, attesta che la stessa istituzione ha

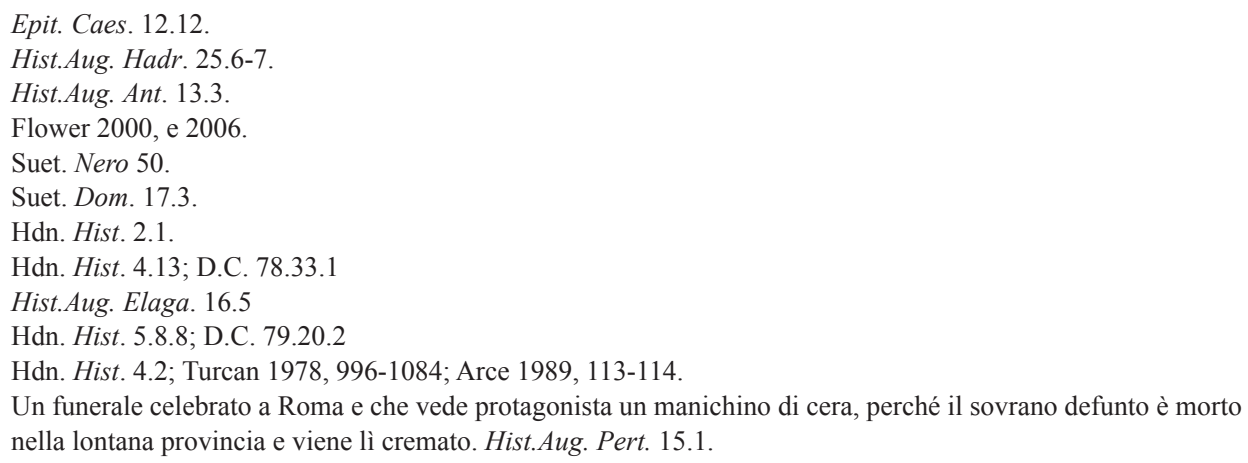


ben presente tale criticità. ${ }^{40}$ Pertanto, deve fornire pregevolezza a quello che deve apparire persino in epoca inoltrata un artificio. Questa esigenza viene sentita in una Roma attaccata ai valori repubblicani, che il Senato custodisce come propria prerogativa. Per questo motivo si percepisce l'urgenza di opporre un contraltare e, pertanto, si deve aumentare esponenzialmente, attraverso la liberazione dell'aquila, il numero dei fruitori di un segno che sta per la realtà che si vuole rappresentare. Tale volo può essere considerato una vera e propria prova dell'imbarazzo in cui si trova il regime nel dover dimostrare puntualmente l'ascesa al cielo dell'imperatore. E se la divinizzazione costituisce un atto di messa in codice di un valore fondante dell'Istituzione, la trappola visiva creata dall'aquila che si libra in cielo sulla pira assurge a finzione parossistica. Difatti, solo nel momento in cui si altera il codice e lo si rende più fruibile, trasportandolo dal piano della speculazione a quello dei sensi, la realtà che si narra può diventare davvero credibile (Fig. 1).

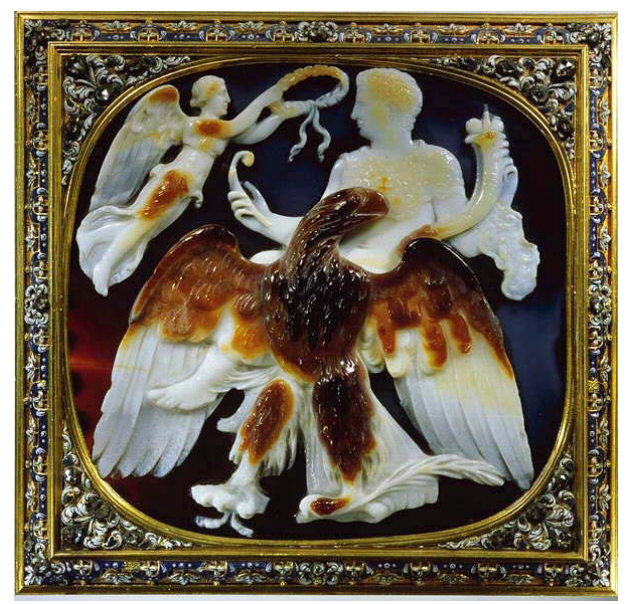

Fig. 1. Claudio ascende al cielo su un'aquila, cammeo in agata e sardonice, Bibliotheque Nationale, Cabinet des Medailles, Parigi, Francia (www.romanoimpero.com).

Sul piano della prassi si osserva come né Erodiano e né Dione attestino l'emissione del senato consulto per Settimio Severo, ciò ha fatto presumere a MacCormack che la consecratio ha a precedere, da un certo punto in poi, il funerale. ${ }^{41}$ Di conseguenza il volo dell'aquila si pone come espressione teleologica, che rende percepibile ai fruitori del cerimoniale il contenuto del decreto senatoriale previamente ottenuto. S'annota che il senato consulto non solo prende atto della diffusione delle idee circa l'ultravita dell'augusto presso un più ampio pubblico, ma costituisce pure un "canale" ufficiale utile all'Impero per esprime le proprie posizioni a riguardo del tema e regolarizzare, quando occorre, l'entusiasmo popolare, che spontaneamente può afferire all'imperatore la divinizzazione..$^{42}$

L'adesione formale di Costantino al cristianesimo ed il progressivo auge della gerarchia ecclesiastica entro la compagine sociale pongono non pochi problemi

Cumont 1910, 119-164, e 1917, 35-118;Cardini 1987, 38-43.

MacCormack 1995, 155.

MacCormack 1995, 155. 
teorici circa la legittimità del culto imperiale, l'attendibilità e la credibilità della consecratio dell'augusto defunto, nonché le modalità con cui questo espediente della propaganda deve essere proposto ai cristiani. La proclamazione a dio di Diocleziano in ragione della Themistes, quale virtù posseduta sin dalla nascita, almeno sul piano speculativo, pone una barriera insormontabile per i cristiani. Al contempo, il culto imperiale integra nel contesto palatino il cerimoniale dell'adoratio, di cui uno dei gesti di maggiore impatto è la proskýnesis; comportamentalità che difficilmente può essere accettata dai cristiani più rigorosi. Eppure è proprio il suo inserimento nel cerimoniale palatino a modificarne progressivamente la percezione, fin tanto da produrre una "laicizzazione" del rito. Cosa che rende lecita la pratica innanzi ad un imperatore cristiano, senza creare troppe contraddizioni con le credenze religiose di un qualsivoglia fedele o dell'imperatore stesso. ${ }^{43}$ Orbene, la problematica sembrerebbe risolversi in un falso problema.

Un processo agevolato dal peculiare contesto in cui si realizza: Arce sostiene che il IV sec. è un "siglo fluctuante durante el que se produce una transformación que, como todas, es lenta y contiene factores retardatarios en el que existen nuevos componentes que ya anuncian o representan el cambio." ${ }^{44}$ Una possibilità di reflussi anche comportamentali, che permette da un certo punto in poi l'accettazione del culto imperiale ai cristiani. Anzi è il cristianesimo, dopo la conversione di Costantino, che con le proprie elaborazioni corrobora il solidificarsi di alcune idee circa l'ultravita dell'augusto. Difatti “neoplatonismo e cristianesimo coincidono nel fare del monoteismo un problema politico, configurando il monarca come unico rappresentante in terra del Dio unico e supremo dell'universo". ${ }^{45}$ Costantino, paradossalmente, appare almeno sul piano della speculazione come il vero erede della 'teologia politica' di Diocleziano. Questi poi è aiutato dai politologi cristiani e soprattutto dal "teologo politico" Eusebio, che elabora una "cristologia" capace di giustificare il culto imperiale, rendendo quell'imperatore partecipe delle qualità della divinità cristiana. Eusebio già nell'orazione dei Tricennalia arriva a sostenere la Christomimesis imperiale, ${ }^{46}$ quale atto di ricezione delle consuetudini pagane circa la mimesi divina dell'augusto entro un'alea di continuità. Credenze che vengono reinterpretate e rifunzionalizzate dalla speculazione cristiana, che fin dai tempi di San Paolo ha sostenuto fortemente la necessità di obbedire al rappresentante del potere costituito e di offrirgli deferenza. ${ }^{47}$

Una percezione diffusa della divinità dell'augusto dunque, che però non sembra convincere gli intellettuali, poiché la ritengono una 'favola' per il volgo. Oltre la patina aulica della propaganda solo gli intellettuali e forse i più scettici del popolo riescono a distinguere il senso reale di concetti sofisticati come la divinizzazione dell'imperatore, il ruolo di vicario della divinità e l'essere la somma magistratura dell'Impero. Al dubbio degli scettici, gli intellettuali sostituiscono la più raffinata dottrina della 'doppia verità'; concetto ben presente ai neoplatonici. Costoro sono consapevoli che esiste una 'verità' da somministrare al volgo ed una che solo gli eruditi custodiscono come privilegio elitario. Possiamo allora ritenere che una di

\footnotetext{
Teja 1993, 616.

Arce 1989, 160.

Teja 1993, 617.

Eus. LC 2-5.

Paul. Ep. Rom. 13.1-7; Ep. ad Tit. 3.1, e Ep. ad Thim. 1.2.1-2.
} 
quelle 'verità' destinate al volgo concerne la natura divina imperiale. Nel privato, invece, gli intellettuali si limitano a considerare la funzione meramente istituzionale del potere esercitato dall'augusto, senza che questa consapevolezza possa entrare in contraddizione con la formula ufficiale, che ne giustifica il culto. ${ }^{48}$

Una formula ufficiale a cui però tutti gli intellettuali aderiscono, perché le elaborazioni culturali del periodo non permettono d'esprimere in pubblico certe verità scomode per l'Istituzione. Anzi le stesse elaborazioni degli intellettuali producono un pacchetto di soluzioni utili a rendere incontrovertibile tale status dell'imperatore.

Eusebio in funzione di "teologo politico" si obera del compito di interpretare il vissuto di Costantino entro il contesto di un panegirico dominato da finalità apologetiche. Un'opera che deve espungere ogni elemento capace di contrastare con l'assoluta santità dell'imperatore e deve consegnarlo definitivamente al cielo. Per questo non desina nell'adoperare lo stratagemma dell'espunzione di fatti e condotte incompatibili col ritratto che propone. Espediente che usa quando non è in grado di interpretare adeguatamente ed in chiave edificante gli avvenimenti. Si limita allora ad un tono vago, con cui allude a certi eventi, per poi trasferire subito l'attenzione su qualche altro accadimento che ritiene più utile alla propria narrazione.

Culmine di tale attività apologetica è l'apoteosi imperiale, che viene narrata in termini filosofici comprensibili ad una corte di fede variegata destinataria del componimento. Opera rivolta pure al figlio Costanzo II, a cui viene indirizzata piuttosto con fini paideutici. La Vita Constantini, stanti le normali manipolazioni dei fatti tipiche del genere biografico e le altrettanto consuete esagerazioni, deve essere valutata come metro per comprendere lo stato delle elaborazioni della dottrina del potere nel periodo di riferimento ed appare utile ad intendere quale possa essere la percezione comune circa le credenze sull'ultravita dell'augusto.

Eusebio viene oberato del compito di testimoniare l'apoteosi imperiale, come ha fatto Numerio Attico per Augusto. Inventa così uno stratagemma: nell'incipit della sua Vita Constantini ha a volgere idealmente gli occhi al cielo per immaginare il destino ultraterreno del Costantino trapassato, sicché "quell'anima (...) -appare- sia unita a Dio in persona, sia libera da ogni involucro mortale e terreno, e splendente di una veste abbagliante di luce". ${ }^{49} \mathrm{Ne}$ fornisce una descrizione accattivante dunque, mentre acclara la sua apoteosi come fatto compiuto. Arriva poi a sostenere che il defunto ha ormai aumentato esponenzialmente le proprie potenzialità. Ma non solo. Può persino affermare, adoperando un'immagine tradizionale della consecratio, che questi sta assiso sull'arco del cielo, da dove contempla però il Dio dei cristiani. La rifunzionalizzazione di elementi della consuetudine pagana diventa così il paradigma dell'operazione posta in atto. Aggiunge poi che questi sembra quasi vivere in simbiosi con la divinità, fin tanto da costituire una sorta di tutt'uno ${ }^{50} \mathrm{ed}$, al contempo, lo vuole presente in ogni luogo dell'Impero. Quale redivivus non soffre tutti gli effetti della morte e, sebbene dimora presso Dio, può essere presente in terra per guidare l'azione dei figli. Il concetto di redivivus per Calderone conforma piuttosto Costantino appena trapassato all'idea tutta cristiana del santo. ${ }^{51} \mathrm{Il}$ vescovo, redattore della biografia costantiniana, diviene così il garante del suo accesso all'ultravita.

\footnotetext{
Teja 1993, 618; a riguardo della dottrina della doppia verità si cita ex multis Sinesio, cfr. Sin. Ep. 80-95, 105.

Eus. $V C$ 1.1.2.

Eus. $V C$ 1.1.2.

Calderone 1972, 262-263.
} 
Un transito che Eusebio evoca anche attraverso la menzione della moneta emessa per la consecratio imperiale, laddove la propaganda della dinastia Flavia ricorre ad un locus classico: quello dell'imperatore che sale al cielo sul currus Solis.52 Una scena che viene completata da un altro topos: la mano divina che scende dall'alto e tende verso di lui, per compiere la dextrarum iunctio (Fig. 2). ${ }^{53}$ Si raffronta una soluzione classica, che si ritrova pure nella Historia Augusta: Settimio Severo sogna di essere portato via da questo carro su un globo, laddove attende la visione di Giove, che lo accoglie fra gli dei..$^{54}$
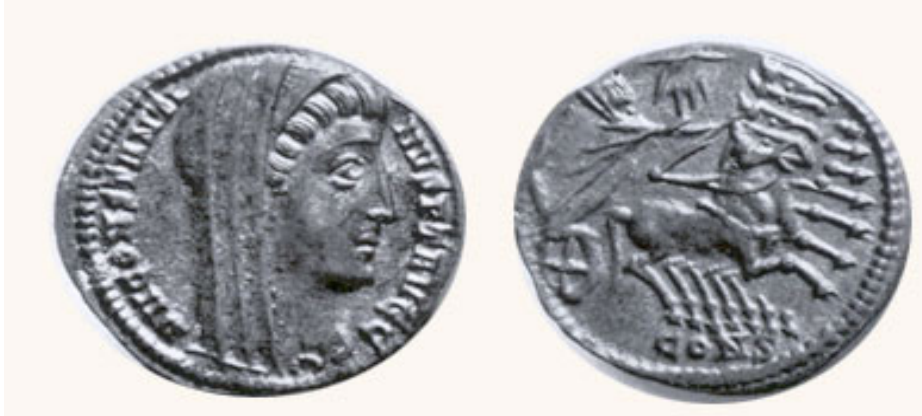

Fig. 2. Moneta commemorativa della dipartita di Costantino, dritto con Costantino col capo velato, retto con Costantino auriga, Zecca di Alessandria (www.monetaecivilta.it).

Ma vi è di più. Eusebio nel descrivere gli ultimi giorni di vita di Costantino rende palpabile la sua ansia di immortalità. La sua ultravita allora non può derivare dall'essere mero 'iniziato' al "mistero della regalità", ma richiede qualcosa di più. Questi deve anche iniziarsi ai misteri della religione cristiana: per questo deve chiedere il battesimo. Pertanto, Eusebio si impegna nel presentare "la cerimonia in chiave mistica (...): questo Battesimo ritardato diventa quasi un'apoteosi cristiana". ${ }^{55}$ Una scelta quella di Costantino, che culmina nel disprezzo della porpora, quale soluzione retorica da riportarsi al locus d'umiltà della tradizione agiografica:

I vescovi eseguirono quanto è prescritto dalla legge divina e compirono i sacri riti (...). Quando il rito si fu concluso, indossò bianche e regali vesti, splendenti al pari della luce, e si sdraiò su un candidissimo letto, né volle più toccare la porpora. Poi, alzando il tono della voce, rese la preghiera di ringraziamento a Dio, aggiungendo subito dopo le seguenti parole: "Ora so di essere beato nel vero senso della parola, ora so di avere meritato la vita eterna, ora so di avere in me la luce eterna". ${ }^{56}$

Siamo di fronte ad un logos sull'immortalità dell'anima, che deve investire il "mistero della regalità" e tutte le sue implicazioni. Alla nuance porpora che isola

52 Eus. VC 4.73; Bruun 1954, 37-48; Koep 1958, 94-104; Barnes 1981; Elliot 1990, 349-353; MacCormack 1995, 178; Caccamo Caltabiano 2002, 31-46.

$53 \quad$ Pan. Lat. 7.7.1

54 Hist.Aug. Sept. 22.1.

55 Marcone 2002, 170; Amerise 2005, 48.

56 Eus. VC 4.63.2, trad. it. Tartaglia 2001, 202. 
l'augusto si sostituiscono le lenzuola e la tunica bianche, che sanciscono lo stato di "nuova creatura", in quanto colore della purezza acquisita col battesimo. ${ }^{57}$

L'elemento chiave per l'accesso tra i celesti dell'imperatore è la sua iniziazione cristiana, pertanto è l'apostasia, in uno alla negazione dei valori di quella religione, che condanna Giuliano imperatore. La fazione pagana tenta comunque di consegnare all'Olimpo l'ultimo degli augusti che aderisce all'antica religione, attraverso la produzione di una serie di elaborazioni fra cui spicca un oracolo trascritto da Eunapio concernente la sua apoteosi. Questi può rappresentare l'ascensione al cielo di Giuliano in termini solari, mentre viene rapito dal carro. ${ }^{58} \mathrm{Ma}$ ciò non sembra bastare. La propaganda cristiana arriva ad additare la morte di Giuliano ad un adynaton e la lancia che gli trafigge il fianco viene scagliata addirittura da San Mercurio. ${ }^{59}$ La leggenda aurea di Basilio Magno allora accoglie un tale motivo: il santo prega la Vergine per la liberazione della Chiesa dall'imperatore apostata, la quale fa resuscitare Mercurio temporaneamente per effettuare tale azione. E se Basilio ottiene il patronato in battaglia contro gli infedeli, Giuliano subisce la definitiva damnatio da parte della Chiesa che ha osteggiato. Una tradizione concernente la forte personalità del vescovo che è credibile dopotutto, perché lo stesso Basilio nel 327 non ha esitato ad accompagnare l'imperatore Valente fuori dalla sua Chiesa. ${ }^{60}$

La fede cristiana può salvare persino un imperatore come Valentiniano II, che muore formalmente suicida e nonostante sia sospetto di arianesimo. Le ragioni politiche spingono Ambrogio vescovo di Milano a salvarne la memoria ed a proclamare nella sua Consolatio de obitu l'apoteosi imperiale. Stante la dottrina della Chiesa sul tema, si considera come il vescovo non solo espunge l'evento dalla narrazione, ma falsifica il racconto, spostando l'attenzione sull'elemento metafisico e sulle idee cristiane circa la vita ultraterrena dell'imperatore. Intanto sulle circostanze della morte fa calare il silenzio. Siamo di fronte ad un'opera di raffinata esegesi, che attraverso l'elemento della selezione, dell'espunzione e dell'adattamento tenta di tradurre nell'idioma cristiano i loci pagani e filosofici dell'ascesa alle sfere celesti, arricchiti con figure bibliche esportate dal Cantico dei Cantici.

Ambrogio allora può immaginare che al suo ingresso in cielo il defunto imperatore venga accolto dal suo pari: Graziano. Elabora pure un supposto dialogo fra i due. Qui Valentiniano non viene riconosciuto come imperatore dal collega, il quale in virtù della comune fede lo apostrofa solamente e gioiosamente: "fratello". Il de obitu per Valentiniano rappresenta allora un tentativo di descrizione della vita ultraterrena riservata all'imperatore. Una traduzione in chiave cristiana della consecratio dunque, che adopera l'immaginario comune per tradurre in immagini compatibili e coerenti con la nuova fede alcune idee connesse all'ascesa dell'augusto ai cieli già fatte proprie dal paganesimo. Immagini che conservano appunto una matrice gentile e filosofica, per essere "trasformate in qualcosa di diverso". ${ }^{61}$ Questo perché il cielo nella concezione cristiana appare come il luogo ove è possibile ottenere la mercede per le opere compiute in vita, il luogo del sollazzo e del riposo, ove si giunge dopo le fatiche terrene:

Sulla veste bianca: Paul. Gal. 3.27; Tert. De Res. 27.1-2; Cypr. De mort. 20; Chyrs. Cath. 4.4.22; Pass. Perp. 4; Cyr. Cath. Myst. 4.8.11.

58 Eun. Hist. frag. 26 c; Nock 1957, 115-123.

59 Jac. Var. Leg. Aur. 250, trad. it. C. Lisi.

60 Gr. Naz. Or. 20.

61 MacCormack 1995, 226. 
Nel momento in cui l'anima salì al cielo Graziano, fratello dell'imperatore, lo vide e l'abbracciò (...)."Vieni, -disse- fratello mio, usciamo all'aperto nei prati e riposiamoci sui colli; svegliamoci all'alba nelle vigne". Ossia, "sei giunto nel luogo dove $\mathrm{i}$ frutti di virtù diverse sono garantiti secondo i meriti di ognuno $\mathrm{e}$ dove, per i meriti, abbondano i premi". ${ }^{62}$

Tale affermazione dimostra che Valentiniano viene assolto dall'imperdonabile peccato del suicidio, sempre se mai avvenuto. L'ascesa al cielo funge da 'clausola di salvaguardia' ed espunge dalla storia la sua condotta filo-ariana.

Ambrogio allora compendia le teorie cristiane sulla vita ultraterrena dell'augusto con quelle pagane e tenta di aggiornare queste ultime. ${ }^{63}$ Crea pure un connubio tra lo status eccezionale dell'imperatore e quello più comune, ma altrettanto essenziale, del fedele. Ma l'essere imperatore implica necessariamente qualcosa in più e presuppone una certezza maggiore circa la propria vita ultraterrena. Perché la salvezza non può essere negata all'augusto senza intaccare la dottrina del potere. Questa 'messa in codice' e la sua falsificazione appaiono necessari alla magnificazione dell'Istituzione ed Ambrogio lo sa. E se Eusebio fa di Costantino un servo di Dio che nutre la speranza di entrare nel regno dei cieli in qualità di fedele, Ambrogio al contrario è costretto dagli accadimenti a recuperare la tradizione. L'imperatore cristiano del resto, come i predecessori pagani, deve accedere al cielo in ragione dell'ufficio svolto. E questo non è un dettaglio secondario. Egli vive nei termini della certezza il potenziale destino di tutti coloro che professano la sua medesima fede.

In occasione del de obitu Theodosii Ambrogio può rimodulare la narrazione e fare della fede cristiana il motivo principe della sua collocazione fra i celesti: "Che cos'è la fede se non la sostanza delle cose in cui speriamo?" ${ }^{64}$ La vita ultraterrena dell'imperatore è pur sempre frutto della sua fede personale; non è solo un corollario di status: "Poiché i giusti vivono grazie alla fede (...)". ${ }^{65}$

Ambrogio certamente entra in contraddizione con quanto espresso per Valentiniano, ma la situazione è cambiata ed alcune cautele non hanno senso d'essere approntate. Cambia anche il luogo di arrivo. Egli non viene ammesso nel giardino, ma giunge alla città imperiale per eccellenza: la Gerusalemme celeste, sede del "Gran Re".

L'immaginario in via di consolidamento permette al vescovo di superare i limiti posti dall'apoteosi raccontata da Eusebio. La gloria di Costantino, oltre ogni sperimentazione, si muove entro gli spazi di copertura imposti dalla filosofia neoplatonica e dalle produzioni artistiche di genere, per non creare spaesamento cognitivo e visivo nel suo pubblico. ${ }^{66}$ Ambrogio invece può proporre nuove soluzioni nella rappresentazione della concezione cristiana della vita ultraterrena dell'imperatore. Reinterpreta così il rapporto fra il defunto, i predecessori che lo aspettano a Gerusalemme, che non è percepita più come "il cuore dei patriarchi, ma il luogo di gloria dei sovrani cristiani” ed i suoi figli; il tutto entro il regime di straordinaria continuità dell'alea della monarchia cristiana. ${ }^{67}$

\footnotetext{
Ambr. De Ob. Valent. 71-72, cfr. trad. it. F. Piviotti Inghilleri, cit. in MacCormack 1995, 226.

Dudden 1935, 417-421.

Ambr. De Ob. Theod. 7.8, cfr. trad. it. F. Piviotti Inghilleri, cit. in MacCormack 1995, 225.

Ambr. De Ob. Theod. 11-12, cfr. trad. it. F. Piviotti Inghilleri, cit. in MacCormack 1995, 225.

Versnel 1970, 123.

MacCormack 1995, 225.
} 
L'introduzione dell'immagine della Gerusalemme celeste ha a ribadire la sacralizzazione della sede imperiale ${ }^{68} \mathrm{E}$ se la presenza dell'augusto, in quanto "divinità imperiale", basta a rendere sacra la capitale terrena, l'opera di Ambrogio crea una rete fitta di rimandi, che esalta il luogo del riposo dei resti mortali degli imperatori. Al contempo, il richiamo alla Gerusalemme del cielo aumenta la sua spendibilità sul piano retorico, in quanto sede perpetua dell'anima imperiale. Si dimostra un'ulteriore messa in codice e l'interpretazione permette lo scivolamento di significato e l'agevole trapasso tra i due significanti. Uno status privilegiato quello dell'imperatore, ribadito dai tituli che assume: paradisi incola ed habitator supernae illius civitatis. ${ }^{69}$ Il benvenuto nella 'città del cielo' gli viene poi tributato dai "principi cristiani" che qui già dimorano, quali Constantino, Elena e Graziano:

Teodosio si affrettò a entrare in quel luogo di riposo; si affrettò a entrare nella città di Gerusalemme, di cui è detto "i re della terra li vivranno la loro gloria". Quella è la vera gloria, portata fin lassù, là quello è riconosciuto come il più beato dei regni e verso il quale si affrettano anche gli apostoli (...) Teodosio degno di venerabile memoria, ora gioisce della luce perpetua e della eterna tranquillità e per le azioni compiute in questo corpo è premiato con i frutti della ricompensa divina. Teodosio, degno di venerabile memoria, merita la compagnia dei santi perché amava il Signore suo Dio. $^{70}$

La sacralizzazione della Respublica Christiana attraverso la glorificazione della fede dei suoi reggenti si consuma in un incontro di gioia con i santi ed "Ora Teodosio degno di venerabile memoria, sa di governare quando si trova nel regno del Signore Gesù".71

Emerge allora un immaginario tutto cristiano che, con le sue soluzioni tutto sommato coerenti, si oppone alla letteratura pagana di genere. Un tentativo insomma di risolvere i diversi contrasti di opinione generati nel lungo periodo dalla riflessione sul tema, che vengono acquietati solo a Bisanzio.

Nel panegirico pronunciato per l'ascesa di Giustino II si giunge persino a collegare l'elezione del nuovo imperatore alle esequie di Giustiniano. Corippo per legittimare ulteriormente l'eletto si preoccupa di descrivere i riti con cui questi consegna ai celesti il proprio predecessore. Per tale ragione la narrazione include un logos sull'immortalità dell'anima, che Giustiniano morente avrebbe pronunciato circa la sua imminente dipartita:

Mi stanno chiamando, amico mio, e il mio ultimo giorno si avvicina; al comando del mio creatore devo lasciare questo mondo per quell'altro (...)-abbandonata la carneil mio spirito sarà passato ascendendo attraverso la volta scintillante del cielo $(\ldots)^{72}$

Al contempo ricorre al locus dell'epifania divina declinato in termini onirici e fa della Gran Madre di Dio, che impone una corona all'imperatore eletto, un

\footnotetext{
MacCormack 1995, 225; Amb. De Ob. Theod. 56.

MacCormack 1995, 225.

Ambr. De Ob. Theod. 31-32, cfr. trad. it. F. Piviotti Inghilleri, cit. in MacCormack 1995, 227.

Ambr. De Ob. Theod. 39-40, cfr. trad. it. F. Piviotti Inghilleri, cit. in MacCormack 1995, 227.

Coripp. Laud. Iust. 4.337-344, trad. it. F. Piviotti Inghilleri, cit. in MacCormack 1995, 236.
} 
incontestabile testimone. Questa rivolgendosi a Giustino nei modi e nei termini della consolatio, rivela l'apoteosi del predecessore:

Perché versi lacrime? Perché piangi sulla felicità? Metti da parte la tristezza, tuo padre vive e vive in cielo e gioisce nel suo grande giorno. ${ }^{73}$

La rappresentazione della Vergine deve fugare ogni dubbio sull'apoteosi di una figura complessa e contestata come quel Giustiniano, che nelle Historia arcana è visto da Procopio muoversi nel palazzo, di notte e senza sonno, come un fantasma. ${ }^{74}$ Lo stesso personaggio che il medesimo autore descrive come il "principe dei demoni" nelle visioni di un santo monaco giunto in visita alla capitale. ${ }^{75}$ Proprio quell'uomo che Procopio paradossalmente loda nel De Aedificiis. Nel cosmo cristiano si ammette persino la possibilità di chiamare a testimoniare la divinità o sua Madre ad affermare l'ultravita dell'augusto nei termini della certezza, perché le elaborazioni culturali del tempo non permettono di poter sostenere il contrario in pubblico, né di predicare l'ateismo apertamente.

\section{Gli attori "coprotagonisti”: la corte ed i partecipanti}

La formale adesione di Costantino al cristianesimo non può non avere conseguenze sul rito funebre. Anche se la sua scelta di fede si inserisce in un momento in cui il cristianesimo è sola religio licita, ma non ancora l'unica religione ufficiale dell'Impero, i riti che consegnano Costantino al sepolcro devono necessariamente tenere conto di questo stato dei fatti. Il cerimoniale allora viene riformulato e conserva alcuni momenti fondamentali come la translatio, la conclamatio, l'expositio e lo iustitium, tutti elementi che non pongono problemi ad essere integrati con i riti funebri cristiani, configurando un precedente utile per il futuro. Si può parlare pertanto di continuità in certi aspetti esteriori, o addirittura del loro consolidamento. Nemmeno pone particolari questioni la volontà di spettacolarizzazione dell'eventologia dell'Impero, tanto che queste esequie possono essere considerate un caso paradigmatico della teatralizzazione posta in essere dalla corte che le organizza. Questa non solo progetta in maniera puntuale la prossemica da inscenare davanti al feretro, ma dirige le azioni dei figuranti prevedendo un percorso preciso da svolgere ed una serie di comportamenti entro una sequenza di scene fissa ed inemendabile.

Tuttavia, coloro che costruiscono la cerimonia devono immaginare un ruolo altrettanto attivo per il popolo, il quale accorre per partecipare e diviene anch'esso un attore "coprotagonista" del rito.

La Vita Constantini fornisce una serie di informazioni utili circa la predisposizione di una precisa prossemica da tenere durante tutto il tempo del lutto. ${ }^{76} \mathrm{Si}$ dimostra che a Costantinopoli viene rifunzionalizzata una prassi già approntata sotto Augusto ed istituzionalizzata sotto i Severi: nonostante l'evento morte tutta la vita di corte si

\footnotetext{
Coripp. Laud. Iust. 1.49-51, trad. it. F. Piviotti Inghilleri, cit. in MacCormack 1995, 236.

Procop. Arc. 12.24-26.

Procop. Arc. 12.20.

Franchi De' Cavalieri 1962, 265-309.
} 
svolge come se l'augusto fosse ancora in vita. Eusebio narra quanto accade attorno alla prothesis:

I generali di tutte le forze armate, i conti e gli altri magistrati, che già da prima erano soliti prostrarsi dinanzi all'imperatore, non mutarono le abitudini loro consuete: entravano (...) nella camera ardente, si inginocchiavano e anche da morto rendevano omaggio all'imperatore giacente nella bara, proprio come se fosse ancora in vita. ${ }^{77}$

Un trucco del protocollo dunque, quello di immaginare l'imperatore vivente, quale soluzione che si pone in continuità con la tradizione. Una prassi che non è legata a mera opportunità, ma ad una meditata volontà di far sopravvivere parte della tradizione romana nel contesto cristiano. Come notato da MacCormack il funus dell'imperatore cristiano con la sua teatralizzazione assume un ruolo fondamentale nella politica di propaganda, perché permette di trasmettere una serie di messaggi concernenti la visione cristiana del potere costituito, incarnata nello status di cui è investito l'imperatore, non solo in vita, ma ancor più nella morte. Specie allorché la Chiesa sembra assicurare la sua presenza innanzi al Dio dei cristiani.

Un discorso veramente incisivo rispetto alle elaborazioni dei politologi gentili, che si limitano ad assicurare un mero destino ultraterreno allo statista che ha esercitato la virtù della giustizia e la buona gestione della cosa pubblica. Una verità che persino uno scettico come Cicerone si preoccupa di perorare nel Somnium Scipionis, entrando in contraddizione con quanto egli stesso ha già detto circa la vita ultraterrena nel finale del Cato Maior e nel De amicitia. ${ }^{78}$ Le elaborazioni rappresentate nel Somnium si concludono entro un contesto filosofico e religioso, che ha un fine paideutico piuttosto. L'opera difatti coltiva, entro i limiti di una visione affabulatrice, l'illusoria pretesa che possa bastare educare la classe dirigente a disprezzare la vanità del potere. Questi arriva persino a sostenere che lo statista non deve confondere il prestigio personale col bene comune. Deve piuttosto volgere le sue aspirazioni ad una ricompensa trascendente e non a cose vane o di poco conto, come il potere temporale. ${ }^{79}$

Simili teorie non possono certo pretendere un qualche perdurare delle funzioni sovrane oltre la morte come fa Eusebio. La soluzione retorica approntata da quest' ultimo cerca di superare le diverse questioni concernenti il dibattito sull'ultravita dell'imperatore, che ha animato la speculazione pagana. Problematiche che in Eusebio vengono risolte col ricorso ad un discorso capace di far suoi alcuni termini filosofici e teologici. Si propongono modalità descrittive della vita ultraterrena che possono essere intese sia dal pagano, sia dal cristiano. Un vocabolario filosofico che si mostra ambiguo, sicché l'espressione $\alpha \psi \tilde{i} \delta \varepsilon \varsigma$ ov̉ $\alpha{ }^{\prime} v i \alpha 1$, non necessariamente pagana, si affianca ad immagini certamente pagane come la citazione del currus Solis e l'evocazione del consessus caelitum ${ }^{80}$ Gli onori riferiti alla salma di Costantino poi sembrano alludere ad un culto, assimilabile a quello che la Chiesa della cronologia

\footnotetext{
Eus. VC 4.67.1, trad. it. Tartaglia 2001, 206.

Cic. De am. 14; Ronconi 1993, 89-90.

Ronconi 1993, 86-87.

Eus. VC 4.69; Karyannopulos 1956, 341-357; MacCormack 1995, 174-175.
} 
di riferimento afferisce ai santi. ${ }^{81}$ Questo perché arriva a sostenere che l'imperatore viene esentato dal giudizio universale per ascendere direttamente al cielo e regnare con Dio; ciò lo solleva dal destino riservato ai comuni cristiani. Una soluzione che pone un particolare problema circa i modi di rappresentazione dell'immortalità dell'imperatore. ${ }^{82} \mathrm{Si}$ arriva così ad affermare una continuità tra vita ultraterrena $\mathrm{e}$ vita terrena dell'augusto, fin tanto da poter dar adito alla credenza che fa considerare il corpo del defunto imperatore ancora senziente. Un espediente sufficiente a suggestionare il lettore, giustificare in chiave cristiana una prassi finalizzata a gestire nel migliore dei modi l'interregno e rafforzare la pretesa di eternità dell'Impero attraverso la concessione dell'immortalità agli augusti.

E se il cerimoniale sembra cercare un equilibrio fra tradizione e nuovo che avanza, si nota come molti etimi della dottrina del potere rimangono sostanzialmente inalterati.

Emerge ancora il ruolo del successore che guida la cerimonia e con i soldati omaggia il defunto comandante in capo dell'esercito. Eusebio così narra i fatti:

Ma quando giunse il secondo dei suoi figli decise di far celebrare in pubblico i funerali del padre, ponendosi egli stesso alla testa del corteo funebre. Precedevano numerosi drappelli di soldati disposti in schiere compatte, seguiva una immensa folla di gente, mentre il corpo dell'imperatore era circondato da lancieri e da opliti. Quando giunsero nella chiesa degli Apostoli del Salvatore, vi deposero la bara. E così il nuovo imperatore Costanzo rese omaggio al padre suo, e con la sua stessa presenza, oltre che con tutte le altre onoranze, compì i doveri richiesti dalla pietà filiale. Quando si fu ritirato con tutti i soldati, si fecero avanti i sacerdoti insieme con un'enorme folla di fedeli, ed iniziarono a recitare le preghiere e a compiere la sacra funzione. Il santo principe giaceva nel tempio su di un alto catafalco e in suo onore avveniva la celebrazione di quel rito: una gran massa di gente, unitamente ai sacerdoti, tra molti lamenti e non senza lacrime, elevava al Signore preghiere per l'anima dell'imperatore, adempiendo così la volontà stessa di quel sovrano amato da Dio. ${ }^{83}$

Qualche dubbio sorge circa la perpetuazione a Costantinopoli della decursio e della rituale dispersione dei dona militaris ${ }^{84}$ questo perché Eusebio concentra piuttosto l'attenzione sul ruolo attivo dei sacerdoti che celebrano il rito funebre e sui canti previsti dalla liturgia, quale caratteristica fondamentale del rito cristiano. Segnala, infine, la partecipazione attiva del popolo che arriva ad offrire lacrime per il defunto, quale massima espressione del consenso raggiunto dall'augusto dipartito. Costanzo, in quanto catecumeno, deve abbandonare la celebrazione nel momento dell'Eucarestia, facendo dei sacerdoti gli 'attori' principali del rito. Preti e coro fungono da 'figuranti' e svolgono un ruolo tanto attivo, quanto coattato: devono ossequiare l'ordo liturgico, ma al contempo hanno a rispettare il protocollo di corte. Ben più liberi appaiono invece gli astanti, che però vengono suggestionati dal cerimoniale approntato, fin

81 MacCormack 1995, 174; Brown 2002. Cfr. Filost. HE 2.17.

82 Eusebio sembra dare per scontata la diretta ascesa al cielo di Costantino e, persino, della madre Elena: Eus. $V C$ 3.46; tale destino oltre all'imperatore sembra essere riservato ai martiri: Chesnut 1986, 157-158.

83 Eus. VC 4.1, trad. it. Tartaglia 2001, 206.

84 Arce 1989, 54-56; Lozano Gómez 2009, 153-173. 
tanto da versare lacrime per il defunto; ciò a riprova dell'ottima orchestrazione. Un dettaglio che non può essere considerato una mera clausola di stile, ma un dato piuttosto verosimile; Costantino non può essere certo additato come un cattivo sovrano, ma tutt'altro. Lo dimostra il fatto che nella lontana Roma viene approntato il classico funerale, forse secondo la formula del funus imaginarium dato che il corpo viene traslato a Costantinopoli. Un cerimoniale che è accompagnato dal consueto iustitium, quale forma di lutto pubblico ${ }^{85} \mathrm{Al}$ contempo, il Senato non esita e si affretta a concedere la consecratio dell'augusto, che si dichiara apertamente cristiano, e lo colloca de plano fra i divi pagani. ${ }^{86}$ Non può essere altrimenti, perché la consecratio al tempo è divenuta un atto dovuto per i buoni sovrani e poco ha a che fare con l'antico diritto senatorio di porre in discussione la condotta dell'imperatore. Un culto tutto pagano dunque, tributato all'augusto dichiaratosi cristiano, che è confermato dal calendario di Filócalo redatto per l'anno 354, laddove si commemora il 27 di gennaio il N(atalis) D(ivi) Constantini, una festa celebrata con 24 corse di carri al circo.

Non mancano però momenti di rottura come l'abolizione della pira, già soppressa da Diocleziano, il quale ritiene inutile ogni atto purificatorio, dato che egli è conspicuus et praesens Iuppiter, ${ }^{87}$ mentre la sua divinità si è già rivelata a sufficienza durante il regno; quindi ad essa nulla si può aggiungere. Un atto rituale andato in disuso anche a causa dell'affermarsi dell'inumazione. Venendo meno la pira, viene anche meno il costume di liberare l'aquila al momento della sua accensione. Le elaborazioni culturali, almeno quelle diffuse fra gli intellettuali, permettono di ritenere superflui questi due elementi fondamentali nel rito funebre imperiale. Due segni che possono essere sostituiti sia dal rito religioso cristiano, perché capace di consegnare al cielo Costantino defunto, sia dal sepolcro di porfido, quale capsula atta a contenere la salma. Il corpo di Costantino, così composto, diviene allora oggetto di venerazione come quello dei santi, fin a costituire una reliquia insigne. Al contempo, si raffronta un espediente utile a reinventare il culto imperiale.

La narrazione della processione funebre che riporta a Costantinopoli il cadavere di Costanzo II fornisce ulteriori dettagli sul ruolo dei figuranti e sulla partecipazione popolare; vi aggiunge persino informazioni sull'irrompere del soprannaturale nel rito. Gregorio di Nazianzo, al di là di una sua personale presa di posizione, seppur autorevole, dimostra che il quadro sociale in cui si celebra il funus è cambiato. Questi può riproporre il motivo 'classico' della familia funesta impersonata dal popolo che attornia il feretro; eppure si preoccupa di reinterpretarlo. Il popolo, venuto meno il valore del giudizio senatorio, col suo spontaneo partecipare alle esequie, emette il verdetto 'definitivo' sulla condotta del proprio imperatore. Gregorio ripropone nell'Impero cristiano l'atavica antitesi fra consecratio e damnatio, presentandola a corollario del cerimoniale funebre. Si reintroduce nei riti funebri quell'espressione articolata di un consenso, che pone in relazione la popolazione e l'imperatore. ${ }^{88}$ Nell'interpretare i fatti Gregorio ha pure presente che il funus imperatorum è connotato da una certa 'flessibilità' prossemica ed è sempre 'pronto' a recepire nuove sollecitazioni, quale sistema aperto a continui 'correttivi'; tutti volti ad ottimizzare le diverse forme di espressione del consenso. Il valore positivo della condotta imperiale

Eus. $V C$ 4.69; 4.70-71, trad. it. Tartaglia 2001, 205-206.

Eus. VC 4.69; 4.70-71, trad. it. Tartaglia 2001, 205-206.

Pan. Lat. 3.10.5.

MacCormack 1995, 191. 
viene ratificato poi dall'adynaton, tanto che per Costanzo II sembrano cantare gli angeli. Subentra allora l'elemento irrazionale, che aumenta l'efficacia della descrizione, rendendola ben più toccante. Il consensus omnium viene supportato dal Cielo ed espresso dalle voci angeliche:

Il corpo di Costanzo venne fatto uscire con solennità e tra acclamazioni generali, accompagnate dalle nostre cerimonie religiose -il cantare per tutta la notte e il fare luce con delle torce- che sono i riti con i quali noi cristiani pensiamo sia giusto onorare una morte santa. L'esposizione del cadavere è quindi un'occasione per una solenne gioia collettiva mescolata al cordoglio (...) e molti hanno anche inteso che, mentre il corpo si trovava a passare attraverso i monti del Tauro per essere riportato nella sua città natale, si fosse sentita una voce dalla cima delle montagne, come di esseri che cantassero e incitassero (Costanzo) a proseguire, la voce, io credo, di potenze angeliche, un premio per la sua pietà e un'offerta per il suo funerale. ${ }^{89}$

L'adynaton allora permette persino di assolvere Costanzo da ogni sospetto di professare l'eresia ariana. Tuttavia tale scelta narrativa nasconde un ulteriore intento, che prescinde dal comportamento coattato dei 'figuranti' della processione e da quello indotto del popolo partecipante. ${ }^{90}$ Infatti siamo di fronte ad una 'messa in codice'. La scelta narrativa serve a giustificare l'inappellabile condanna di Giuliano. Non è un caso che l'affectio del popolo e dei soldati costringano Giuliano, il quale nell'attesa fieramente ostenta le imperialia insignia sulle soglie di Costantinopoli, a privarsene per assumere le vesti del lutto al fine di scortarlo ai Santi Apostoli. ${ }^{91}$ Nonostante ciò, sappiamo che Giuliano organizza per il cugino un funerale tradizionale, differenziato soltanto dall'inumazione nel mausoleo cristiano.

Anche in questo caso, oltre le velleità della fonte cristiana che interpreta i fatti, emerge il ruolo dei figuranti: corte ed esercito sono guidati dal successore che è il più interessato ad ottenere una cerimonia funebre senza tumulti e capace di coagulare il consenso su di sé. Compare ancora il popolo che è coinvolto dal "Teatro del potere", non solo nel partecipare, ma persino nel mostrare sentimenti mesti. Un'induzione, quella alla compartecipazione, che è la finalità ultima di un rituale che ordisce la trama delle prossemiche possibili.

Anche Ambrogio di Milano ha a porre l'attenzione sul ruolo dei partecipanti al funus imperatorum, mentre commemora la dipartita di Teodosio con la pronuncia di un'orazione funebre. Per ottimizzare il messaggio da trasmettere sceglie di ricorrere ad un locus ben presente al suo pubblico: 1'adventus.92 Questi si preoccupa così di evocare non solo la massiccia partecipazione popolare, ma persino quella soprannaturale alle esequie di Teodosio. Sicché il corteggio umano non appare sufficiente ad onorare il buon imperatore cristiano. Pertanto, trasfigura in melius il protocollo ed arriva addirittura ad affermare che si aggiungono alle armate imperiali a fargli da scorta nel suo ultimo viaggio anche le angelorum catervae e la sanctorum turba in qualità di comitiva imperiale. Questi spiega: la compagnia angelica che accompagna il cadavere di Teodosio in terra e la sua anima in cielo è obbligata

Gr. Naz. PG 35.685 ab; cfr. trad. it. F. Piviotti Inghilleri, cit. in MacCormack 1995, 190.

MacCormack 1972, 721-752.

Filost. HE 6.6; Soc. 3; Amm. Marc. 21.16.21; Dagron 1991, 35.

MacCormack 1972, 721-752. 
a tale compito dalla sua conversione al cristianesimo e dalla sua fede in Cristo. Di conseguenza afferma: “dove invece c'è fede, l'esercito è composto da schiere di angeli". ${ }^{93}$ La proposizione di un codice tutto cristiano presuppone un pubblico avvezzo a questo tipo di soluzione o, comunque, in grado di comprenderla.

La sublimazione dell'adventus non permette al narratore di prescindere dai loci di vittoria. L'opera obbliga al ricorso ai termini del repertorio del trionfo. Ambrogio si appoggia ad una tradizione consolidata e ridefinisce l'evento morte, tabuizzando la dimensione luttuosa ed interpretando i fatti alla luce della semantica della Vittoria. E se già Seneca ha adoperato tale soluzione, ${ }^{94}$ Ambrogio la riempie di colori cristiani, collegandola piuttosto alla singolare vittoria sulla morte dei martiri. Una novità nel panorama della fenomenologia della regalità, che il vescovo non può introdurre senza sapere di avere un pubblico pronto a recepire la formula. Siamo di fronte ad un'operazione consapevole di somministrazione di codici votati al successo. ${ }^{95}$ È difatti il locus della Vittoria che ottimizza la ricezione della nuova soluzione interpretativa, mentre la teologia offre formule altrettanto valide per la rappresentazione della vita ultraterrena dell'imperatore. Si ha quindi coscienza dell'esistenza di un certo patrimonio immateriale, sempre utile alla 'messa in codice' dei fatti, che sopravvive nonostante la svolta cristiana. ${ }^{96}$

La fede dell'augusto permette di sublimare le istanze del protocollo, potenziandolo fino al limite estremo: questo può dunque coinvolgere gli angeli ed i santi, che vengono a partecipare alla cerimonia funebre e scortano il cadavere imperiale. Il dettaglio, oltre la forza affabulatoria della soluzione in sé, ci offre la misura del potere persuasivo fatto proprio da questo cerimoniale. E Ambrogio lo sa bene. Questi si volge ad utilizzare soluzioni comunque condivise, poiché ha presente che adoperando una formula non aderente all'immaginario del suo auditorio, non solo va incontro al rischio di non convincere il pubblico, ma può persino incorrere nell'inefficacia del componimento.

Il rito d'esequie approntato nel 656 alla morte di Giustiniano e descritto da Corippo permette di apprezzare l'estrema raffinatezza nella progettazione delle prossemiche che i figuranti devono tenere. Un'organizzazione minuziosa, che enfatizza il dolore e la mestizia: vi sono difatti dei piangenti che circondano la salma. Un dolore che viene contrapposto allo splendore della prothesis, messa in opera come una qual sorta di 'trionfo', che prevede l'ostentazione dei mirabilia populi romani. Difatti si collocano nei pressi del catafalco, oltre al prezioso drappo che avvolge il corpo ed a molti candelabri di metallo prezioso, tutte le gioie più belle del tesoro imperiale.

La 'macchina' di corte in seguito pone in essere la pompa funeraria, che Corippo racconta con gusto narrativo per il dettaglio prezioso. Giustino, i rappresentanti dei Demi ed alcuni tra i senatori sorreggono candelabri d'oro e d'argento con candele accese e si recano con suggestiva movenza presso il catafalco, altri partecipanti bruciano incenso; intanto i preti e le suore intonano canti. La nuova imperatrice, Sofia, offre una veste color porpora con ricami in oro rappresentanti i trionfi del defunto; tale dono fa luce su un'altra funzione della veste: il "dono pietoso". ${ }^{97}$

Ambr. De Ob. Theod. 10, cfr. trad. it. F. Piviotti Inghilleri, cit. in MacCormack 1995, 225.

Sen. Cons. ad Marc. 3.1.

MacCormack 1995, 225.

MacCormack 1995, 225.

Ravegnani 1984; 1989, 160, e 2008. 
Corippo si preoccupa di riferire la grande affluenza del popolo costantinopolitano, che si assiepa per vedere la processione funebre divenendone un partecipante: "Uomini e donne di ogni età uscivano per strada. Chi avrebbe potuto narrare le meraviglie di una tale processione?". ${ }^{98}$ La pompa si conclude alla chiesa dei SS. Apostoli, laddove Giustiniano viene deposto in un sarcofago d'ostrite verde. ${ }^{99}$

Il meraviglioso diventa allora fonte di suggestione ed è capace di fare del popolo un partecipante, richiamandolo con lo stupore delle risorse devolute all'effimero; lo induce poi a comportamenti mesti. L'opulenza diventa, allora, cifra peculiare nell'interazione e fa del partecipante un "coprotagonista" del rito.

\section{Il palcoscenico: i SS. Apostoli ed il culmine del funus}

Il funus imperatorum, che coinvolge la città con la processione funeraria, congiunge idealmente il Sacro Palazzo dove si allestisce la prothesis al luogo di sepoltura: l'Apostoleion con gli annessi mausolei imperiali.

Dal punto di vista geografico la chiesa dei SS. Apostoli, si ubica nel tratto settentrionale della Mese, fra il Philadelphion e la porta di Carisio. ${ }^{100}$ L'edificio ha una parabola di vita complessa, di cui si prende in considerazione solo la facies fra IV e V secc.

La prima fase di vita del complesso, che risale al IV sec. pone molteplici questioni di cui si considerano le due più pregnanti: la controversa identità del fondatore $\mathrm{e}$ la dibattuta morfologia planimetrica dell'edificio. Una problematica, quella della fondazione, che costituisce un corollario della "questione costantiniana", dato che le fonti non sono concordi nell'identificare il vero fondatore.

$\mathrm{Si}$ deve necessariamente procedere da quanto riferisce Eusebio sulla progettazione e destinazione dell'edificio per volontà di Costantino al culto degli apostoli ed a proprio sepolcro:

L'imperatore consacrò l'intero complesso allo scopo di perpetuare in eterno il ricordo degli apostoli del nostro Salvatore. Ma nell'edificarlo perseguiva dentro di sé anche un altro intento (...). Egli, infatti, intese riservare a sé stesso quel luogo per quanto fosse giunto il momento della sua fine, preoccupandosi con uno straordinario atto di fede di fare in modo che il suo corpo dopo la morte venisse accostato e accomunato al nome degli apostoli: sperava di beneficiare così anche dopo la morte delle preghiere che colà si sarebbero recitate in onore degli apostoli. ${ }^{101}$

Dal racconto si deduce che la chiesa è da subito destinata al culto cristiano e l'imperatore la fornisce di sacerdoti, che offrono preghiere in suo favore agli apostoli e per questo: "dispose che in quel tempio si tenessero anche delle pubbliche funzioni, e a tale scopo fece installare un altare proprio al centro dell'edificio". ${ }^{102}$ Descrive ancora la predisposizione della tomba imperiale:

\footnotetext{
Coripp. Laud. Iust. 1.130; 170-180; 208.

Bekker 1843, 203-207.

Dagron 1991, 393.

Eus. $V C$ 4.60.1-2, trad. it. Tartaglia 2001, 199.

102 Eus. VC 4.60.2, trad. it. Tartaglia 2001, 199.
} 
fece erigere dodici sarcofaghi, alla stregua di sacre steli in onore e memoria della comunione degli apostoli; nel mezzo fece collocare la cassa che era destinata a sé stesso, in modo che questa avesse su ciascun dei due lati sei dei sarcofaghi consacrati agli apostoli. ${ }^{103}$

E se certa dottrina ha voluto liquidare il passo come spurio, non è però riuscita a fornire alcuna prova filologica o stilistica a riguardo. ${ }^{104} \mathrm{~A}$ parere di chi scrive appare coerente con lo speculum delineato da Eusebio l'opera di un pio imperatore, che si assicura preghiere dopo la morte e vuole favorire la benevolenza degli apostoli per il proprio trapasso. Il vescovo suggerisce un indizio utile a delineare meglio la personalità di Costantino. Indizio che tra l'altro si ritrova anche negli scrittori pagani. Emerge sommessamente il ritratto di un uomo che si riconosce peccatore, quale contraltare dell'uomo tormentato delineato da Zosimo ed Eutropio. ${ }^{105}$ Questi, comunque sia, cerca la salvezza e la trova nella dottrina di Cristo e ciò a prescindere dal dato suggerito da Zosimo: i riti eleusini espiativi dopo la riforma neoplatonica non appaiono più adeguati ad assolverlo dalla colpa d'aver condannato a morte il figlio e la seconda moglie. ${ }^{106}$

Altre fonti confermano la paternità costantiniana, quali Paolino di Nola, Teofane, Glica, Cedreno, Giorgio Monaco, Socrate, Sozomeno ed i Patria. Queste non solo si limitano ad additare a Costantino la progettazione dell'edificio, ma ripropongono un evidente anacronismo e si porta in scena finanche la madre Elena. ${ }^{107} \mathrm{Al}$ contrario Filostorgio menziona una stanza rotonda nettamente distinta dalla chiesa, che Mesarite definisce heroon. ${ }^{108}$ Mentre Procopio, Costantino Rodio, Teodoro il Lettore e Nicola Mesarite citano Costanzo II quale fondatore. ${ }^{109}$

Eppure il vescovo di Cesarea narra i funerali e la deposizione all'interno della chiesa, cosa che pare un nonsense se la si ritiene non finita. Nondimeno, lo stesso Eusebio avrebbe dovuto sopportare l'imbarazzo del sostenere una così imprudente manipolazione della storia a poco tempo dai fatti. Con buona probabilità Filostorgio è caduto in un equivoco ed ha confuso l'erede-promotore dei funerali ed il fondatore dell'edificio. Questo anche se Mesarite sostiene che Costantino sia stato sepolto fin dalla sua morte in S. Acacio e non solo in via provvisoria dal 359 d.C. circa. ${ }^{110}$

La prima fase di vita dell'edificio è 'fotografata' da Eusebio, anche se questi ci fornisce una ricostruzione generica e poco dettagliata, riferendo meramente l'esistenza di un soffitto dorato a cassettoni, della presenza del marmo che sembra rivestire le pareti dell'edificio fino al soffitto e di una copertura di bronzo dorato. ${ }^{111}$ Offre ancora un'informazione importante: la chiesa è collocata all'interno di un quadriportico su cui si affacciano i presunti appartamenti riservati agli imperatori, i bagni e gli alloggi dei guardiani. Oltre ogni prosaicità di Eusebio, si deve considerare

\footnotetext{
Eus. VC 4.60.3, trad. it. Tartaglia 2001, 199.

104 Downey 1951, 61; Krautheimer 1983, 224.

Zos. 2.29; Iul. El. Const. 6; Eutr. 10.6.3-7.

106 Zos. 2.29; vedi anche: Iul. Caes. 336 A-C. Questa tesi viene puntualmente demolita nel sec. V da Sozomeno, cfr. Soz. 2.5 .

107 Downey 1951, 53-80. Si citano ex plurimis: Eus. VC 4.58-60; Paulin. Carm. 19; Soc. 1.16-40; Soz. 2.34.

108 Nic. Mes. 1.39.

109 Filost. HE 2.2; Procop. Aed. 1.4.19; Theod. Lect. PG 86, col. 213; Nic. Mes. 1.39.

110 Soc. 2.38; Soz. 4.21; Janin 1969, 18.

111 Eus. VC 4.58-59, trad it. Tartaglia 2001, 198.
} 
la necessità di provvedere in modo adeguato alla fabbrica che deve contenere non il corpo di un semplice imperatore, ma costituire il sacrario del fondatore della stessa città.

L'aula sembrerebbe essere a forma di croce greca, come si evince dal Carmen de insomnio Anastasiae di Gregorio Nazianzeno, ove viene descritto all'incrocio delle braccia il cosiddetto $\delta \omega \mu \alpha \tau_{\tau} \tau o v .{ }^{12}$ Uno spazio adatto ad accogliere il "cenotafio" degli Apostoli, il $\lambda \alpha \dot{\rho} \rho v \alpha \xi$ (sepolcro) di Costantino e le $\vartheta \tilde{\eta} v \kappa \alpha$ dei dodici apostoli, disposte attorno alla sua tomba, forse a semicerchio o quarto di cerchio, con una precisa allusione al progetto della tomba gerosolimitana di Cristo. Eppure non si comprende

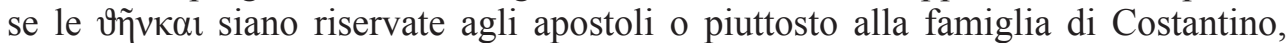
facendo della chiesa un mausoleo dinastico.

Seguendo Gregorio Nazianzeno si può postulare l'esistenza di un atrium o di un peribolo rettangolare con un quadriportico, nel cui mezzo è ubicata la chiesa vera e propria. Sull'atrio sembra che si affaccino poi gli oĩ Koเ $\beta \alpha \sigma i ́ \lambda \varepsilon$ เol, corpi di fabbrica intesi come appartamenti imperiali, data la distanza considerevole fra la chiesa-mausoleo ed il Sacro Palazzo. Tale interpretazione è resa più plausibile se si considera che nelle altre capitali della Tetrarchia, come le città di Tessalonica e Spalato, il palazzo imperiale prevede in sua prossimità il mausoleo. ${ }^{113}$ Secondo un ulteriore interpretazione tutte le pertinenze sembrano destinate piuttosto ai soli custodi (Fig. 3).

Mango concilia i dati evinti dalle fonti e sostiene la costruzione di un semplice mausoleo imperiale sotto Costantino, a cui si affianca la chiesa cristiana per volontà

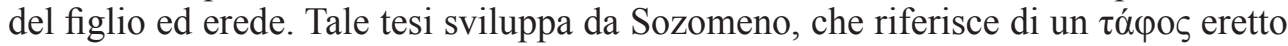
da Costantino, mentre Costanzo II ordina la fondazione dei SS. Apostoli. ${ }^{114}$

Giovanni Crisostomo poi riferisce la decisione presa da Costanzo II, volta a collocare la tomba del padre in un mausoleo esterno, direttamente comunicante con la basilica, denominato "anticamera del pescatore", perché "suo figlio ha ritenuto di fare grande onore a Costantino il grande collocandolo nel vestibolo del pescatore", quasi a far da "portiere" agli apostoli. ${ }^{115}$

Suscita particolare attenzione l'allocazione del $\lambda \alpha \alpha_{\rho} v \alpha \xi$ imperiale entro la configurazione del palcoscenico di questo "Teatro del potere". Una sede che viene opportunamente allocata fra i dodici monumenti riservati agli apostoli. Siamo di fronte ad un'evidenza probatoria della "religione di Costantino", che lo fa identificare quale "tredicesimo dio" pagano, nel luogo di un culto degli dodici dei olimpici, come suggerito da Pseudo-Callistene. ${ }^{116}$ Eppure il sepolcro dell'imperatore collocato al centro del consesso apostolico si oppone al sepolcro di Cristo in Gerusalemme, la "città di Cristo" e la capitale cristiana dell'Impero. ${ }^{117}$ Una contrapposizione che si realizza anche nelle assonanze della planimetria e diventa una prova della pretesa Christomimesis imperiale, già teorizzata nell'orazione per i Tricennalia. ${ }^{118} \mathrm{Un}$ parallelo che fa del sacrario costantinopolitano il santuario della resurrezione e

\footnotetext{
Gr. Naz. Car. de insom. An., PG 37, 1258, vv. 59-60.

Krautheimer 1983, 38; Dagron 1991, 91.

Mango 1985, 51-62; Soz. 2.34.

Chrys. Hom. in Ep. 2 Cor. 26.4, PG 61, coll. 580-582.

Ps.Callisth. HA 8.35; Downey 1951, 59, nota 25.

Dagron 1991, 412-414.

118 Eus. $L C 2-5$.
} 
dell'apoteosi della "divinità imperiale". ${ }^{119}$ Un santuario collocato in una città che di lì a poco le tante reliquie accumulate trasformano nella "nuova" Gerusalemme, come la vita di S. Daniele stilita la ridefinisce. ${ }^{120}$

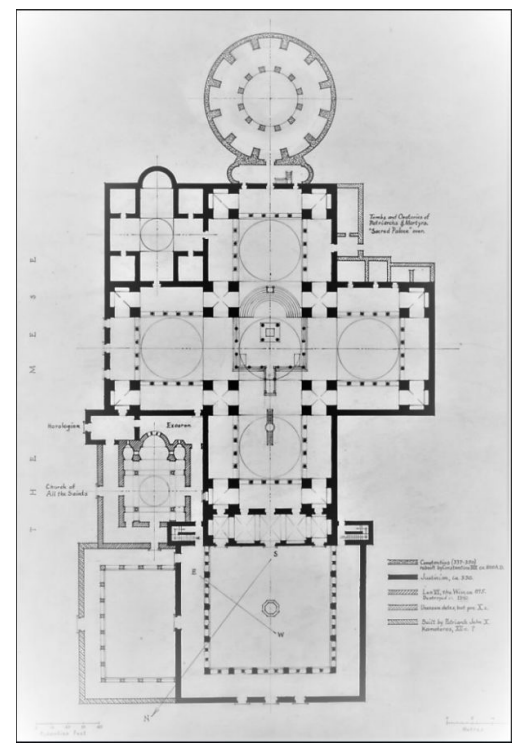

Fig. 3. Ricostruzione della pianta giustinianea della chiesa dei SS. Apostoli (Paul Underwood 1948, ICFA, MS.BZ.019-BF.F.1993.F2825).

Si raffronta una sorta di 'trionfo' riservato a Costantino ed una magniloquente scenografia, sia se situata all'intersecarsi delle "braccia" della croce, sia se addossata all'abside. Il passo di Eusebio non lascia intendere se questo sia allocato presso l'altare o se costituisca esso stesso un altare o, ancora, se prenda il posto della cattedra. E se l'assenza di ogni preciso riferimento all'esatta ubicazione dell'altare fa sospettare l'esistenza di una locazione defilata dall'aula liturgica, la menzione della sinassi eucaristica fa credere piuttosto che questa sostituisse l'altare. ${ }^{121} \mathrm{Se}$ si ritiene che l'edificio, consacrato per Girolamo nel 370 d.C., ${ }^{122}$ sia stato dedicato al culto imperiale, non stupisce che la tomba possa costituire un vero pedante all'altare, annoverando il corpo imperiale fra le reliquie insigni, per lo meno fino all'arrivo dei resti mortali dei veri santi: l'apostolo Andrea, l'evangelista Luca e S. Timoteo; un evento attestato nel 338 d.C. secondo Paolino da Nola. ${ }^{123}$

Una forma di culto imperiale così palese difficilmente può essere accettata dalla Chiesa, tanto che Costanzo II per evitare nuove tensioni con la gerarchia ritira la tomba dall'aula e la colloca in un mausoleo rotondo: l'anticamera del pescatore ricordata da Giovanni Crisostomo. Eppure il tema di Costantino "uguale agli apostoli" non compare solo nella Vita Constantini di Eusebio, ma diviene persino

\footnotetext{
119 Piganiol 1932, 241-242; Grabar 1946, 252-253.

120 Vit. S. Dan. stil. 11-12.

121 Heisenberg 1908, 100-101.

122 Hieron. Chron. 356-357.

123 Paulin. Carm. 19, vv. 329-342.
} 
un leitmotiv della propaganda del sec. V. Compare anche in Gregorio Nazianzeno, il quale afferma che "la gloriosa schiera degli apostoli ha accolto e protegge la famiglia sacra, la quale gode degli onori quasi uguali a loro"; ${ }^{124}$ si ricuce la frattura fra culto imperiale e pratica ecclesiastica e si fa salva la destinazione del mausoleo dinastico.

Nel 359 d.C. secondo la Notizia di Costantinopoli, allorché l'Apostoleion si rivela pericolante, il patriarca Macedonio trasferisce la bara di Costantino presso la chiesa di Sant'Acacio di Caria, la più vicina ai Santi Apostoli. ${ }^{25}$ Evento che suscita il pubblico tumulto.

Più tardi, mentr'è regnante Giustiniano, la chiesa costantiniana viene rasa al suolo, forse perché fatiscente, per essere così riedificata. Il progetto viene affidato agli architetti Antemio di Tralle ed Isidoro di Mileto, i medesimi progettisti della S. Sofia. Una chiesa che secondo lo Pseudo-Codino viene riedificata per ordine di Teodora.

La nuova fabbrica prevede una pianta a croce libera capace di suggestionare Costantino Rodio, le cui braccia di eguale misura le fonti precisano siano orientate geograficamente. La costruzione prevede a dire di Nicola Mesarite il significativo numero di 12 pilastri e 70 colonne, con chiaro riferimento al numero degli apostoli e a quello dei discepoli di Cristo riportato nei Vangeli. ${ }^{126}$ Colonne che per Costantino Rodio sono 48 per il piano inferiore e altre 48 , che precisa di marmo rosato, per il loggiato superiore; vi è pure la presenza di alcune colonne di marmo frigio sempre a dire dello stesso Costantino, mentre i pilastri sono di pietra di Docimio. Si annoverano ben cinque logge che si dispongono attorno al santuario e circondano il perimetro della chiesa, fino ad incorniciare l'abside. Il pavimento è di marmo colorato, probabilmente di colori tenui, mentre l'alzato è diviso in tre sezioni separate da cornici; sezioni composte di lastre quadrangolari di marmi pregiati. Marmi policromi che Costantino Rodio puntualmente enuclea: lastre bianche e rosse tendenti al porpora dalla Caria, dalla Galazia quelle che hanno per il poeta il "colore della cera" e altre ancora bianche dall'Eritrea; marmi verdi dalla Tessaglia e la pietra cosiddetta serpentina dalla Libia; si aggiungono il porfido egiziano e la sardonica dall'India; nonché quelle provenienti da località più vicine come il marmo proconesio o di Paro.

La struttura è servita da un nartece ed un esonartece, sormontata poi da cinque cupole, una centrale molto grande che sembra assimilabile a quella di S. Sophia, ma solo più piccola, ed altre quattro minori site sui bracci della croce. Una cupola, quella centrale, che ai tempi di Nicola Mesarite è decorata da una rappresentazione del Cristo Pantokrator. Un'immagine dipinta, parte di un più ampio ciclo, che comprende sempre per Mesarite mosaici a fondo d'oro, secondo un programma rinnovato sotto Basilio I alla fine della crisi iconoclasta.

Il santuario, definito "tabernacolo" da Procopio, viene collocato all'intersecarsi delle braccia, quasi a formare una "valva". ${ }^{127}$ Qui si trova la cattedra corredata di synthronon, mentre l'altare in materiale prezioso che accoglie le spoglie di Luca, Andrea e Timoteo è sito sotto un ciborio quadrato, sormontato da una copertura piramidale in porfido. Nell'aula sono custoditi anche altri corpi santi: nel braccio

124 Gr. Naz. Contr. Iul. 2, Or. 5.17, PG 35, coll. 685-688.

125 Soc. 2.38; Soz. 4.21; Downey 1951, 56; Dagron 1995, 339.

126 Const. Rhod. SS. App. vv. 440-452.

127 Procop. Aed. 4.12-17. 
nord viene allocata la tomba di Giovanni Crisostomo, mentre quella di Gregorio il Teologo si trova ad ovest.

Per percepire meglio le spazialità si ricorda l'importante informazione fornita da Procopio di Cesarea nel De Aedificiis, il quale riferisce che la pianta dell'Apostoleion è assimilabile ad un'altra fondazione giustinianea: la chiesa di S. Giovanni in Efeso, risalente al 548 d.C. (Fig. 4). ${ }^{128}$

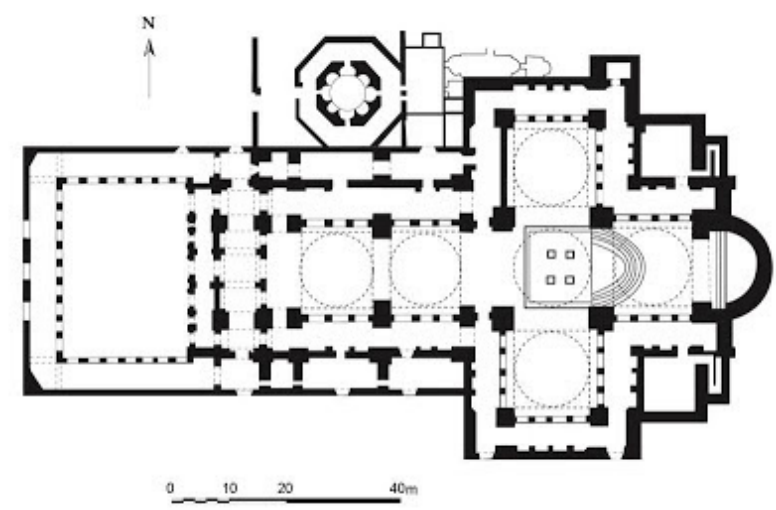

Fig. 4. Pianta della chiesa di S. Giovanni in Efeso (Mango 1978, fig. 162).

La maggior parte dei sepolcri imperiali si concentrano intorno a tombe "mitiche", collocate negli heroa che prendono il nome dei loro più illustri 'inquilini' e promotori, Costantino il Grande e Giustiniano I. I sepolcri degli altri sovrani che fino al 1028 sono inumati nei differenti heroa non sembrano seguire nessun criterio cronologico, ma la devozione dei sovrani verso i predecessori, la volontà di autorappresentarsi come continuatori delle loro gesta, o semplicemente l'opportunità in ragione dello spazio restante. Non deve meravigliare la presenza in questi sacrari delle madri, delle mogli o delle figlie degli imperatori. Una presenza giustificata dall'innato 'ottimismo' bizantino, che la Legge Sallica non conosce, laddove gli uomini ricevono il locus Augusti in via femminile ed in ragione del matrimonio con le esponenti della domus imperialis.

L'heroon di Costantino si ubica alle spalle del santuario, con la sua pianta circolare provvista di cupola e montata su pilastri come Nicola Mesarite attesta. Il corpo di fabbrica secondo il de caerimoniis vanta la presenza di ben diciassette sepolcri, mentre per il de officiis, più tardo, risultano ben diciannove. Qui, oltre alla presenza dei grandi imperatori del Tardoantico, si constata la monopolizzazione degli esponenti della dinastia macedone. Per quel che riguarda i sovrani della cronologia oggetto d'analisi si afferma: il fondatore Costantino sta collocato insieme alla madre Elena a est, mentre il figlio Costanzo II a sud e Teodosio I a Nord, sepolto con la moglie Flaccilla come precisa il Chronicon Altinate; ${ }^{129}$ tutti inumati nel porfido. Anche Marciano e Pulcheria sono consegnati al porfido. Leone I giace in una cassa di marmo verde di ieracite, mentre Anastasio ed Ariadne vengono

${ }_{28}$ Procop. Aed. 5.1.6.

129 Chron.Alt., MGH, supp. 14.62, 
inumati in un sarcofago di pietra nicetiana. Zenone invece ha una tomba in marmo verde di Tessaglia. ${ }^{130}$ Il Chronicon Altinate riferisce pure la controversa sepoltura di Valentiniano I e della moglie Marina Severa in un sepolcro di porfido. ${ }^{131}$

L'Apostoleion è anche il luogo di sepoltura dei patriarchi della città, che si collocano al riparo delle corti porticate che cingono i lati sud e nord dell'edificio. In un padiglione posto a nord vi è il sepolcro di Teodosio II, in un padiglione a sud la tomba di Arcadio ed a est quella di Eudossia. Padiglioni che Zonaras definisce "stoà", ma che per Downey possono indicare piuttosto mausolei privati. ${ }^{132}$ Lo stoà che circonda i lati del tempio custodisce pure i sarcofagi dei principi morti in tenera età. Il Necrologium imperatorum o Catalogus sepulcrorum, composto dall'imperatore Porfirogenito Costantino VII e confluito nel de caerimoniis, riferisce l'allocazione della tomba di Giuliano e della moglie Charito: "In questo portico, che si trova a settentrione, giace un sarcofago dalla forma cilindrica in cui riposa il maledetto ed esecrabile corpo dell'apostata Giuliano, in un sacello dal colore della porpora (...)". Accanto a quel sarcofago sta seppellito Gioviano, tumulato anche questi nel porfido. ${ }^{133}$

Il mausoleo di Giustiniano sta situato al termine del braccio posto a nord dell'edificio: qui le fonti contano sin a ventitré sarcofagi. Tuttavia per la cronologia di riferimento s'annoverano, oltre al fondatore composto in un sepolcro di ostrite e alla sposa Teodora in una cassa di marmo verde di Gerapoli, i successori Giustino II e Sofia in tombe di marmo proconesio. ${ }^{134}$

\section{Gli oggetti di scena: la veste ed il sepolcro di porfido}

Il funus imperatorum nei secoli III-V come ogni forma di spettacolarizzazione richiede una serie di "oggetti di scena", che devono ottimizzare gli effetti affabulatori della cerimonia e migliorare la trasmissione dei messaggi. Fra questi i due più significativi, almeno per l'impatto visuale, possono essere identificati nella veste funebre dell' augusto e, da Diocleziano in poi, nel sepolcro in cui l'imperatore viene tumulato. ${ }^{135}$

Nonostante l'effettivo impatto della veste nel rito, quale mezzo utile a garantire il decoro della salma imperiale, i dati evincibili dalle fonti non permettono di ricostruire per ogni singolo funerale la qualità dell'abito funebre e la quantità dei decori utilizzati. La ricostruzione dell' abbigliamento adoperato durante il funerale di Stato richiede un qualche salto nei passaggi temporali e la collazione di fonti diverse, al fine di rispettare le peculiarità diacroniche del fenomeno. ${ }^{136}$ La penuria di dati è spesso condizionata dalle contingenze e dalle velleità di coloro che narrano l'evento morte.

Aseguito della morte diCostantino e più in generale dal quarto secolo, gli intellettuali cristiani posti a servizio dell' imperatore producono una serie di elaborazioni e pongono

\footnotetext{
Const. Por. Lib. caer. 2.43; Downey 1957; Daskas 2011, 108-111..

Chron.Alt., MGH, supp. 14.62.

32 Zonar. 13.4.28; Downey 1957, 45.

133 Const. Por. Lib. caer. 2.43; Bekker 1943, 207. Tale sepolcro potrebbe contenere però il corpo di Crispo e non certo quello di Giuliano. A tal riguardo si veda: Woods 2006, 364-371.

134 Const. Por. Lib. caer. 2.43; Daskas 2011, 108-111.

135 Turcan 1958; MacCormack 1995, 158-159.

136 Arce 1989, 160.
} 
mano alla riformulazione dell'intero apparato del funus imperatorum. Il risultato di tale azione porta ad un cerimoniale profondamente modellato sulle istanze cristiane concernenti l'ultravita. Tuttavia tale ideologia non sembra investire alcuni aspetti esteriori concernenti la teatralizzazione di questo 'momento forte', quale espediente utile ad ottimizzare il dialogo tra Istituzione imperiale e sudditi. Alla luce di questa esigenza comunicativa, volta ad imprimere nell'inconscio dei sottoposti una precisa idea della maestà, si comprende come la ricchezza dell'abbigliamento continua ad essere una costante delle esequie dei sovrani. Il pensiero cristiano, che predica la sobrietà, sembra perciò aver influenzato davvero poco alcuni aspetti materiali del costume funerario. Le esigenze di spettacolarizzazione sono difatti una priorità nella messa in scena del funus imperatorum.

Le notizie più significative sulla qualità dell'abito funebre per la cronologia di riferimento le troviamo nella descrizione del funus costantiniano. Le velleità agiografiche di Eusebio spingono a descrivere una rinuncia della porpora da parte del Costantino morituro, perché la santità personale lo richiede, mentre il prototipo di una santità d'officio lo reclama.

La corte, tuttavia, non può rischiare lo spaesamento visivo dei partecipanti al funerale. Né può fomentare una qualsivoglia "nostalgia per il paganesimo"137 a seguito del tradimento delle aspettative di un pubblico che non è tutto cristiano. La salma, pertanto, viene composta secondo il cerimoniale consueto. Il rifiuto delle insegne di Costantino viene ignorato e la 'macchina' del protocollo pone in essere strategie di rappresentazione del tutto conformi all' aspettativa sociale. La monarchia non può tradire se stessa e deve fornire ad un ampio pubblico quel novero di segni che la magnificano, la raccontano e la rendono riconoscibile. Siamo di fronte ad un passo indietro della corte rispetto alle esigenze di quel santo che santifica il potere imperiale. La marcata ostentazione delle insegne e l'attenzione al dettaglio prezioso confermano l'esistenza di valori condivisi e posti alla base delle strategie di autorappresentazione dell'Impero romano.

Eusebio fa attenzione a tale dettaglio:

I soldati sollevarono il corpo e lo deposero in una bara d'oro massiccio, che ricoprirono con la porpora imperiale; dopo di che lo trasportarono in Costantinopoli. Qui lo deposero su di un alto catafalco nel salone principale del palazzo imperiale $(\ldots) .^{138}$

Le insegne con cui il corpo viene abbigliato sembrano suggerire l'idea che l'imperatore sia ancora in vita ed additano nell'augusto defunto la già citata figura del redivivus, tabuizzando gli effetti della morte. ${ }^{139}$ Il valore della veste è potenziato proprio dall'attenzione adoperata per descrivere la preziosità dei materiali con cui viene composto il cadavere, perché utili ad ottimizzare la messa in scena. Eusebio addirittura si concentra sulla scelta dei colori, come la porpora di cui è tinta la coperta che orna la salma, nonché sui dettagli preziosi come il materiale della bara. Entrambi segni che qualificano lo status imperiale a mezzo dell'allusione cromatica alla sfera dell'abbondanza. La puntuale menzione di una

\footnotetext{
37 Arce 1989, 160; MacCormack 1995, 171-175.

138 Eus. VC 4.69.1, trad. it. Tartaglia 2001, 70-71.

139 Franchi De' Cavalieri 1962, 265-309.
} 
tale esibizione di fasto da prova del ruolo svolto dall'ostentazione dell'opulenza nel contesto dello 'spettacolo del potere' montato per il funus. Dimostra pure come i fruitori del rito siano attenti al dettaglio, quale cifra peculiare delle strategie di autorappresentazione dell'Impero. ${ }^{140}$ L'abbondanza funge insomma da 'clausola materiale di garanzia' del destino ultraterreno dell'imperatore. Emerge una percezione condivisa da redattore e lettori: è proprio l'abito a fare gli imperatori. Senza tale convinzione dei partecipanti al funus la necessità di un simile dispendio di risorse si risolverebbe in un nonsense.

Una fonte narrativa altrettanto dettagliata si ritrova solo per le esequie di Giustiniano. La 'macchina' di corte mette in scena la prothesis, mentre la salma è adornata di un vestito di porpora e del diadema, allorché è esposta nel Sacro Palazzo, dove riceve l'estremo addio:

Furono lasciate a Giustiniano le grandi insegne che aveva portato da vivo, e, sebbene ormai morto, egli conservava il colorito della vita. La sua nobile morte dimostrò con chiarezza che egli aveva conquistato il mondo. Tra coloro che si trovavano nella camera ardente solo lui sembrava, per il suo aspetto da santo, essere lieto: il suo capo era ornato da un diadema e vedendolo giacere nel letto, ricoperto dalla porpora, era possibile pensare che il suo corpo stesse riposando nel sonno e non nella crudele morte. ${ }^{141}$

L'offerta della veste mortuaria diviene poi occasione per una riflessione sull'universo simbolico e da il pretesto a Corippo per un'ampia descrizione:

Sofia portò il drappo funebre tessuto di porpora preziosa./ Dove tutte le opere di Giustiniano/ Erano rappresentate in oro puro e scintillavano di gemme:/ da un lato c'erano le schiere di barbari prostrati./ L'artista con l'ago esperto aveva composto/ Immagini di re defunti e di nazioni sottomesse (...)./ E nel mezzo del palazzo aveva rappresentato il vincitore in persona./ Nell'atto di schiacciare sotto i piedi il capo del tiranno vandalo,/ Libia lo acclamava e gli offriva frutti e l'alloro:/ L'artista aveva aggiunto Roma con le braccia aperte./ E l'abito che ne mostrava il seno nudo,/ Nutrimento dell'impero e progenitore della libertà./ La saggezza in persona ordinava che fossero fatte queste cose/ In modo che la sua ultima ora potesse condurre Giustiniano/ Alla sua tomba imperiale adorno dei suoi trionfi $(\ldots) .{ }^{142}$

Occorre riflettere sulla funzione gnoseologica di tale veste funerea: i suoi ricami riassumono la vicenda terrena dell'imperatore e spiegano per immagini la "Teologia della Vittoria". La sua descrizione elaborata retoricamente prova che il pubblico, almeno quello a cui il componimento è destinato, è in grado di percepire gli inputs della dottrina del potere di cui l'abbigliamento diviene vettore. ${ }^{143}$ Si può parlare allora di efficacia scenica della veste. Il componimento lascia così vivere nel contesto del funus imperatorum cristianizzato tutte quelle reminiscenze pagane connesse al

\footnotetext{
140 Bourdieu 1983; Flügel 1987; Carile 2000, 65-124, Barthes 2006.

141 Coripp. Laud. Iust. 1.236-247, cfr. trad. it. F. Piviotti Inghilleri, cit. in MacCormack 1995, 233.

142 Coripp. Laud. Iust. 1.276-281; 285-293, cfr. trad. it. F. Piviotti Inghilleri, cit. in MacCormack 1995, 234.

143 McCormick 1986
} 
rito del trionfo, perché adeguate ad un'efficace rappresentazione dell'Istituzione imperiale.

Un altro efficacissimo oggetto di scena viene individuato nel sepolcro a cui è riservato il fasto del porfido, quale privilegio imperiale. ${ }^{144}$ L'impiego di questa pietra egiziana di color rosso scuro e screziata di bianco viene associato da Claudio alle produzioni imperiali, fin tanto che ne assume il valore di status symbol, come Plinio attesta. ${ }^{145}$ Diocleziano invece introduce un'altra novità nei "riti di espulsione" dalla società dell'augusto: l'inumazione del cadavere imperiale in un sepolcro fatto di questo marmo. La pietra viene associata al velamen purpureum, ${ }^{146}$ un panno posto a coprire il sepolcro, che riconduce la pietra a corollario della "liturgia della porpora". ${ }^{147}$

Tuttavia non appare del tutto chiara la ragione che spinge dopo il regno di Marciano a non adoperare più il porfido per i sepolcri imperiali. Anche perché la conquista dell'Egitto da parte degli arabi, che ha fatto venire meno all'Impero l'accesso diretto alle cave di Djebel Dukhan ed al Mons Porphyreticus, si realizza ben oltre i 150 anni dalla morte di quest'ultimo.

Dopo la caduta di Costantinopoli e la distruzione dell'Apostoleion, i sarcofagi imperiali vengono dispersi. Sopravvive però il 'mito' del sepolcro costantiniano e si formano diverse tradizioni circa l'identificazione dell'occupante delle evidenze di porfido afferibili alla nostra cronologia di riferimento. In tal modo si dimostra il valore di questi oggetti, che si estende nel lungo periodo e non si limita al perdurare del tempo concluso del funus. A ciò segue la rifunzionalizzazione di alcuni sepolcri, mentre altri possono subire l'abbandono o addirittura fornire materiale di riuso, come quello proveniente dal Serraglio, che ha permesso di ricostruire ben due sarcofagi.

Sulla scorta dell'emozione alcune fonti prodotte a seguito della caduta di Costantinopoli tendono a stigmatizzare l'empietà dei conquistatori della città, che non rispettano nemmeno i morti e lasciano che il presunto sepolcro di Costantino venga abbandonato a sé, in un luogo indegno non solo allo stato di imperatore, ma indecoroso per qualsiasi defunto. Una realtà che apre all'interpretazione, costituendo una messa in codice ed un espediente critico. Nicolas de Nicolay racconta lo stato deplorevole del presunto sepolcro del fondatore, collocato in una data fra il 1551 ed il 1553 in "una delle strade più luride della città". ${ }^{148}$ Informazione confermata da Jean Palerme che vede in una via squallida, presso il Bazar dei sellai, una tomba di porfido lunga dieci palmi e mezzo, larga sei ed alta sempre sei palmi, che riconosce come la sepoltura di Costantino. ${ }^{149}$ Una collocazione che viene confermata da un altro viaggiatore francese del sedicesimo secolo: Pierre Gylli. ${ }^{150}$ Quest'individuazione si basa su una tradizione che per Hobhouse sembra avere almeno tre secoli. ${ }^{151}$ Un sepolcro da identificarsi con quello ritrovato nel 1910 presso la Colonna di Marciano, in una località molto prossima all'Apostoleion.

\footnotetext{
144 Herklotz 1985.

145 Plin. $H N 36.7$.

146 Amm. Marc. 16.8.4; Arce 1990, 104.

147 Cavallo 1996, 295; Carile 2002, 243-269.

148 Silvius 1576, 94.

149 Pillehotte 1606, 383.

150 Ebersolt 1919, 81; 106.

151 Hobhouse 1817, 350.
} 
Una notizia ben più recente, risalente al 1832, riferisce di una presunta tomba di Costantino, "un sepolcro molto grande (...) -che- potrebbe contenere venti eroi" ornata di fiori, chiusa in un chiosco e venerata come quella dei sultani, presso il cortile della moschea di Bayezid. ${ }^{152}$ Si nota che col passare del tempo la memoria si modifica e non si ha più interesse a far sopravvivere il locus retorico dell'empietà dei nuovi padroni della città. La presunta tomba di Costantino può essere collocata in un luogo decoroso e addirittura venerato dai musulmani.

Un altro sepolcro in porfido, usato come contenitore per l'acqua piovana, viene visto poco tempo prima da Hobhouse presso la moschea di Osman; anche questo viene identificato con la bara di Costantino. ${ }^{153}$

Occorre ancora segnalare che un'ulteriore evidenza funeraria fornita di coperchio, allocata presso la chiesa di Santa Irene, viene indicata dalla dottrina come il sepolcro di Costantino. La formula arcaica del coperchio a tetto "spiovente", più tipica della tradizione romana, rafforza tale presunzione. Un altro efficace indizio lo fornisce Delbrueck, che nel 1932 mappa i fori presenti sul lato del coperchio e dimostra come quelli siano disposti per apporre una croce in metallo di forma patriarcale. Questa è infissa sicuramente postuma, dato che non compare nell'iconografia imperiale prima del sec. VIII. Sulla faccia laterale i fori rilevati si dispongono secondo uno schema che suggerisce la presenza di appliques, tali da far pensare ad un decoro a forma di ruota solare; segno che ricorda quella che in dottrina è ritenuta la prima soluzione grafica adoperata per descrivere il Chrismon. Altrettanto verosimilmente la decorazione può essere interpretata come un'immagine stilizzata del sole con tredici raggi. Un inequivoco indizio della destinazione in favore di Costantino e della sua politica simbolica che si nutre di reflussi segnici.

L'esistenza di decori in metallo rimanda a quanto riferisce Niceta Coniata, che descrive il coperchio tutto "intrecciato d'oro" del sarcofago costantiniano, che è il solo ornamento risparmiato da Alessio III Angelo nell'espoliazione del sepolcreto del 1197 d.C. Sul lato corto del sarcofago si trova scolpita una croce ansata, che adatta l'ankh egiziano unendolo al Chrismon. Un simbolo confacente alla manovalanza egizia, che procede alla produzione di un semilavorato rifinito sicuramente a Costantinopoli. Un simbolo che potrebbe essere letto persino come una reductio del labaro. Un segno che per Piganiol suscita grande suggestione ed evoca l'unione di paganesimo e cristianesimo. ${ }^{154}$ Questo sarcofago è pure dotato di un'epigrafe che viene datata per ragioni paleografiche al IV sec.; ciò a conferma della plausibilità dell'ipotesi (Fig. 5). ${ }^{155}$

\section{Conclusioni}

Il "Teatro del potere" inscenato per le esequie imperiali vuole rendere percepibile ai partecipanti al rito il transito di natura dell'imperatore. Una spettacolarizzazione atta a sancire il momento dell'acquisizione di quello che potremmo definire il

\footnotetext{
Lamartine 1835, 293.

153 Hobhouse 1817, 350.

154 Piganiol 1932, 241-242.

155 Vasiliev 1948, 23.
} 
"corpo politico" e "perpetuo" riservato all'iniziato al "mistero della regalità" e, da un certo punto in poi, all'iniziato al cristianesimo. Eppure la costruzione di questo spettacolo prevede, oltre all'augusto defunto in funzione di protagonista, una serie di figuranti che insieme ai partecipanti al rito fungono da attori "coprotagonisti". Tutti si muovono entro il grande palcoscenico urbano ed entro una processione che culmina al sacrario dell'Apostoleion.

La volontà di garantire l'onore della salma dell'augusto defunto, da cui nessuno può aspettarsi più nulla, attraverso riti adeguati non è null'altro che un tentativo di protezione del decoro dell'Impero, in un momento di rottura, laddove l'Istituzione imperiale può sperimentare la propria debolezza. Una doppia tutela dunque. Il funus imperatorum, quale strumento di 'copertura' istituzionale, conseguentemente diviene oggetto di continuo aggiornamento, in modo da poter rispondere nel miglior modo possibile alle esigenze poste dalle condizioni politiche in cui si verifica l'evento morte.

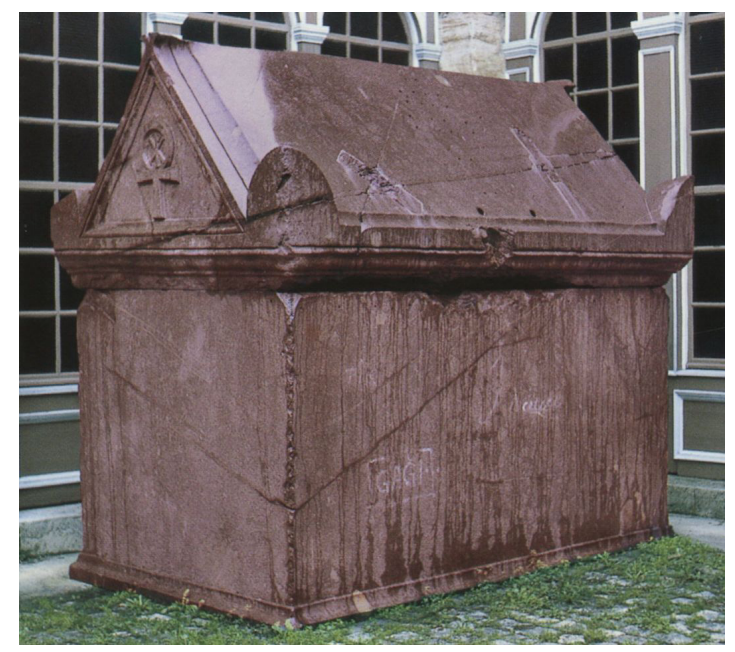

Fig. 5. Sepolcro in porfido proveniente da Hagia Eirene, Archaeological Museum, Istanbul (www.pinterest.it).

\section{Bibliografia}

Alvar, J. (1999): "Religión, política y cohesión social: el culto al emperador”, [in] J. Alvar et alii (ed.), Historia del mundo clásico a través de sus textos, Madrid, vol. II, 272-280.

Alvar, J. - Lozano, F. (2008): "Un tonto entre los dioses: vilipendio del monarca", Potestas 1, 35-56 (http://dx.doi.org/10.6035/Potestas).

Amerise, M. (ed.), (2005): Eusebio di Cesarea, Elogio di Costantino, Milano.

Arce, J.

(1989): Funus imperatorum: los funerales de los emperadores romanos, Madrid.

(2000a): "Muerte, consecratio y triunfo del emperador Trajano", [in] J. González (ed.), Trajano. Emperador de Roma. Actas del Congreso Internacional 14-17 septiembre 1998 (=“L'Erma" di Bretschneider. Saggi di storia antica 16), Roma, 55-70.

(2000b): Memoria de los antepasados: puesta en escena y desarrollo del elogio fúnebre romano, Madrid. 
Barnes, T. D. (1981): Constantine and Eusebius, Cambridge.

Barthes, R. (2006): The Language of Fashion, Oxford.

Beard, M. (2008): El triunfo romano: una historia de Roma a través de la celebración de sus victorias, Barcelona (http://dx.doi.org/10.5860/choice.45-6936).

Bekker, I. (ed.), (1943): Georgii Codini Excepta de antiquitatibus Costantinopolitanis, Bonn.

Bickermann, E. (1929): "Die römische Kaiserapotheose", Archiv für Religionswissenschaft 27, 1-31.

den Boer, W. (ed.), (1972): Le Culte des Souverains dans l'Empire Romain (=Entretiens sur l'Antiquité classique XIX), Vandoeuvres-Genève.

Bourdieu, P. (1983): La distinzione. Critica sociale del gusto, Bologna.

Brown, P. (2002): Il culto dei santi. L'origine e la diffusione di una nuova religiosità, Torino.

Bruun, P. (1954): "The Consecration Coins of Constantine the Great", Arctos 1, 37-48.

Caccamo Caltabiano, M. (2002): "Il simbolismo cosmico della corsa con la quadriga. Temporalità e iconografia del potere", [in] Il simbolo cosmico della quadriga, Tempo sacro e tempo profano, Visione laica e visione cristiana del tempo e della storia, Atti del Convegno di studi (Messina 5-7 settembre 2000), Messina, 31-46.

Calderone, S. (1972): "Teologia politica, successione dinastica e consecratio in età costantiniana", [in] den Boer (ed.), 1972, 215-261.

Cardini, F. (1987): “L'aquila”, Abstracta 13, 38-43.

Carile, A.

(2000): "Le insegne del potere a Bisanzio", [in] La corona e i simboli del potere, Rimini, 65-124.

(2002): "Produzione e usi della porpora nell'impero bizantino", [in] A. Carile (ed.), Immagine e realtà nel mondo bizantino, Bologna, 243-269.

(2003): "La prossemica del potere. Spazi e distanze nei cerimoniali di corte", [in] Uomo e spazio nell'Alto Medioevo, L Settimana di Studio del Centro Italiano di Studi sull'Alto Medioevo, Spoleto.

Cavallo, G. (1996): "La potenza della porpora”, Il Sole 24 Ore, 295.

Cerfaux, L. - Tondriau, J. (1957): Le culte des souverains dans la civilisation greco-romaine (=Bibliothèque de théologie: Théologie biblique 5), Paris.

Chesnut, G. F. (1986): The First Christian Histories: Eusebius, Socrates, Sozomen, Theodoret, and Evagrius (=Théologie historique 46), Macon.

Clauss, M. (1999): Kaiser und Gott: Herrscherkult im Römischen Reich, Stuttgart-Leipzig (http://dx.doi.org/10.1515/9783110956962).

Coarelli, F.

(1983): “Il Pantheon, l'apoteosi di Augusto e l'apoteosi di Romolo, Città e architettura nella Roma imperiale", Analecta Romana Instituti Danici, Suppl. 10, 41-46.

(1985): Il Foro romano, II, Roma.

Crook, J. A. (1996): "Augustus: Power, authority, achievement”, [in] A. K. Bowman - E. Champlin - A. Lintott (eds.), Cambridge Ancient History, Cambridge, 113-146 (http:// dx.doi.org/10.1017/CHOL9780521264303.004).

Cumont, F.

(1910): “L'aigle funéraire des Syriens et l'apothéose des Empereurs", Revue d'Histoire et Littérature Religieuse 42, 119-164.

(1917): "L'aigle funéraire d'Hiérapolis et l'apothéose des empereurs", [in] F. Cumont, Études syriennes, Paris, 35-118.

Dagron, G. (1991): Costantinopoli. Nascita di una capitale (330-451), Torino (trad. it. A. Serafini). 
Daskas, B. (2011): Nicola Mesarite. Descrizione della chiesa dei Santi Apostoli a Costantinopoli. Introduzione, traduzione e commento, Tesi di dottorato, Università di Milano (http://dx.doi.org/10.13130/daskas-beatrice_phd2013-03-22).

Downey, G.

(1951): "The Builder of the original Church of the Apostles at Constantinople. A Contribution to the Criticism of the Vita Constantini attributed to Eusebius", Dumbarton Oaks Papers 6, 51-80 (http://dx.doi.org/10.2307/1291083).

(1958): "The Tombs of Byzantine Emperors at the Church of the Holy Apostles in Constantinople", Journal of Hellenic Studies 79, 27-51 (http://dx.doi.org/10.2307/627920).

Downey, G. (ed.), (1957): Nikolaos Mesarites, Description of the Church of the Holy Apostles at Constantinople, Philadelphia (http://dx.doi.org/10.2307/1005721).

Dudden, F. H. (1935): The life and Times of St. Ambrose, Oxford, 417-421.

Ebersolt, J. (1919): Constantinople byzantine et les voyageurs du Levant, Paris.

Elliot, T. G. (1990): “The Language of Constantine's Propaganda", Transactions of the American Philological Association 120, 349-353 (http://dx.doi.org/10.2307/283996).

Flower, H. I.

(2000): “Damnatio Memoriae and Epigraphy", [in] E. R. Varner (ed.), From Caligula to Constantine. Tyranny and Transformation in Roman Portraiture, Atlanta, 58-69.

(2006): The Art of Forgetting: Disgrace and Oblivion in Roman Political Culture, Chapel Hill (http://dx.doi.org/10.5149/9780807877463_flower).

Flügel, J. C. (1987): Psicologia dell'abbigliamento, Milano.

Franchi De' Cavalieri, P. (1962): "I funerali ed il sepolcro di Costantino Magno", Scritti agiografici 1, 265-309.

Fraschetti, A. (2005): Roma e il principe, Roma-Bari.

Gordon, R. (2001): “The Roman imperial cult and the question of power", [in] L. Golden (ed.), Raising the Eye- brow: John Onians and World Art Studies. An Album Amicorum in His Honour, Oxford, 107-122.

Grabar, A. (1946): Martyrium. Recherches sur le culte des reliques et l'art chrétien antique, Paris, 2 voll.

Kantorowicz, E. (1989): I due corpi del Re. L’idea di regalità nella teologia politica medievale, Torino.

Karayannopulos, J. (1956): "Konstantin der Große und der Kaiserkult”, Historia 5, 341-357.

Koep, L. (1958): "Die Konsekrationsmünzen Kaiser Konstantins und ihre religionspolitische Bedeutung", Jahrbuch für Antike und Christentum 1, 94-104.

Krautheimer, R. (1983): Three Christian Capitals: Topography and Politics (=Una's lectures 4), Berkeley-Los Angeles.

Heisenberg, A. (1908): Grabeskirche und Apostelkirche, Leipzig.

Herklotz, I. (1985): «Sepulcra» e «monumenta» del medioevo. Studi sull'arte sepolcrale in Italia, Roma.

Hobhouse, J. C. (ed.), (1817): A Journey through Albania and other provinces of Turkey in Europe and Asia, to Constantinople, During the years 1809 and 1810, Philadelphia.

Huntington, R.-Metcalf, P. (1979): Celebrations of Death, Cambrige (https://doi.org/10.1017/ CBO9780511803178).

Lamartine, A. de (ed.), (1835): Souvenirs, impressions, pensèes et paysages pendant un voyage en Orient (1832-1833), ou Note d'un voyager, III, Paris.

Lozano Gómez, F.

(2002): La religión del poder. El culto imperial en Atenas en época de Augusto y los emperadores Julio-Claudios (=BAR International Series), Oxford. 
(2007): "Divi Augusti and Theoi Sebastoi: Roman Initiatives and Greek Answers", The Classical Quarterly 57/1, 139-152 (http://dx.doi.org/10.1017/S0009838807000134).

(2009): "El Más Allá de los emperadores: entre la divinización y el olvido", [in] E. Ferrer - F. Lozano - J. Mazuelos (eds.), Salvación, infierno, olvido. Escatología en el mundo antiguo (=Spal Monografías XIV), Sevilla, 153-174.

MacCormack, S. G.

(1972): "Change and Continuity in Late Antiquity. The Ceremony of Adventus", Historia 21, 721-752.

(1995): Arte e cerimoniale nell'antichità (=Biblioteca di storia dell'arte 23), Torino.

Mango, C.

(1978): Architettura bizantina, Milano.

(1985): Le développement urbain de Constantinople (IVE - VII siècles), Paris.

Marcone, A. (2002): Pagano e cristiano. Vita e mito di Costantino, Roma-Bari.

McCormick, M. (1986): Eternal Victory, Triumphal Rulership in late Antiquity, Byzantium and the early Medieval West (=Past and Present Publications 24), Cambridge-Paris (http://dx.doi.org/10.2307/1863561).

Nock, A. D. (1957): “Deification and Julian”, Journal of Roman Studies 47, 115-123 (http:// dx.doi.org/10.2307/298574).

Nogales, T. - González, J. (eds.), (2007): Culto imperial: política y poder (=“L'Erma” di Bretschneider. Hispania Antigua, Serie Arqueológica 1), Roma.

Panascià, M. (ed.), (1993): Costantino Porfirogenito, Il libro delle cerimonie, Palermo.

Parisi, R. (2010): "Immagini di festa", [in] Resta (ed.), 2010, 105-133.

Perea Yébenes, S. (2005): "Imago imperatoris, ad sidera! El funeral de los emperadores romanos, la apoteosis y el "cuerpo doble", Oppidum 1, 103-120.

Piganiol, A. (1932): L’Empereur Constantin, Paris.

Pillehotte, J. (ed.), (1606): De pérégrinations du S. Jean Palerme Forosien, Lyon.

Price, S. R. F. (1984): Rituals and Power. The Roman Imperial Cult in Asia Minor, Cambridge.

Ravegnani, G.

(1984): La corte di Bisanzio, Ravenna.

(1989): La corte di Giustiniano, Roma.

(2008): Imperatori di Bisanzio, Bologna.

Reiske, J. J. (ed.), (1829): Constantinus Porphyrogenitus, Liber de caerimonis aulae byzantinae, Bonn.

Resta, P. (2010): “Introduzione”, [in] Resta (ed.), 2010, 21-45.

Resta, P. (ed.), (2010): Belle da vedere (=Imagines 5), Milano.

Richard, J. C.

(1966a): “Enée, Romulus, César et les funérailles impériales", Mélanges de l'École française de Rome 78/1, 67-78 (http://dx.doi.org/10.3406/mefr.1966.7509).

(1966b): "Les aspects militaires des funérailles impériales", Mélanges de l'École française de Rome 78/2, 313-325 (http://dx.doi.org/10.3406/mefr.1966.7521).

(1966c): "Incinération et inhumation aux funérailles impériales. Histoire du rituel de l'apothéose pendant le haut-empire", Latomus 25, 784-804.

(1966d): "Tombeaux des empereurs et temples des 'divi': notes sur la signification religieuse des sépultures impériales à Rome”, Revue de l'Histoire des Religions 170, 127-142 (http://dx.doi.org/10.3406/rhr.1966.8411).

(1978): "Recherches sur certains aspects du culte impérial: Les funérailles des empereurs

Romains aux deux premiers siècles de notre ère", $A N R W$ II, 16/2, 1121-1134.

Ronconi, A. (1993): Il problema del Somnium Scipionis, Milano. 
Silvius, W. (ed.), (1576): Nicolas de Nicolay. Les navigations, peregrinations et voyages, faicts en la Turquie, Anvers.

Tartaglia, L. (ed.), (2001): Eusebio di Cesarea, Vita di Costantino (=Quaderni di Koinonia 8), Napoli.

Taylor, L. R. (1931): The Divinity of the Roman Emperor (=Philological Monographs 1), Middletown.

Teja, R. (1993): "Il cerimoniale imperiale", [in] A. Carandini et alii (eds.), Storia di Roma, III. L'età Tardo Antica, Torino, 613-642.

Toynbee, J. M. C. (1971): Death and Burial in the Roman World, Baltimore-London.

Turcan, R.

(1958): "Dionysos Dimorphos. Une illustration de la théologie de Bacchus dans l'art funéraire", Mélanges de l'École française de Rome 70, 243-293 (http://dx.doi. org/10.3406/mefr.1958.7431).

(1978): "Le culte impérial au III" siècle", $A N R W$ II, 16/2, 996-1084.

Vasiliev, A. A. (1948): "Imperial porphyry sarcophagi in Constantinople", Dumbarton Oaks Papers 4, 1-26 (http://dx.doi.org/10.2307/1291047).

Versnel, H. S. (1970): Triumphus: An Inquiry into the Origin, Development and Meaning of the Roman Triumph, Leiden.

Wesch-Klein, G. (1993): Funus publicum, Stuttgart.

Woods, D. (2006): "On the Alleged Reburial of Julian the Apostate at Constantinople", Byzantion 76, 364-371. 